\title{
A NEW APPROXIMATE MATHEMATICAL MODEL FOR GLOBAL CONVERGENCE FOR A COEFFICIENT INVERSE PROBLEM WITH BACKSCATTERING DATA
}

\author{
LARISA BEILINA* AND MICHAEL V. KLIBANOV †
}

\begin{abstract}
An approximately globally convergent numerical method for a 3d Coefficient Inverse Problem for a hyperbolic equation with backscattering data is presented. A new approximate mathematical model is presented. An approximation is used only on the first iteration and amounts to the truncation of a certain asymptotic series. A significantly new element of the convergence analysis is that the so-called "tail functions" are estimated. Numerical results in $2 \mathrm{~d}$ and $3 \mathrm{~d}$ cases are presented, including the one for a quite heterogeneous medium.
\end{abstract}

1. Introduction. In this paper we work with a Multidimensional Coefficient Inverse Problem (MCIP) for a hyperbolic PDE with the data resulting from a single measurement event. This means that the data are generated by either a single location of the point source or by a single direction of the incident plane wave. These MCIPs are non-overdetermined ones. For example, in military applications the best way is to collect backscattering data resulting from a single measurement event. This is because an installation of each new source means a life treating risk on the battlefield.

Even though MCIPs have been studied by many researchers since 1960-ies, the topic of reliable numerical methods for them is still in its infancy. This is because of enormous challenges one inevitably faces when trying to study this topic. Those challenges are caused by two factors combined: nonlinearity and ill-posedness of MCIPs. In the case of single measurement the third complicating factor is the minimal amount of available information. It is well known that conventional least squares Tikhonov functionals for MCIPs suffer from the phenomenon of multiple local minima and ravines. Hence, to minimize such a functional, one should apply a locally convergent numerical method, such as, e.g. Newton-like or gradient-like method. Convergence of such algorithms can be guaranteed only if the starting point of iterations is located in a sufficiently small neighborhood of the exact solution. However, the case when a good approximation about the solution is known in advance is rare in real applications.

In a series of recent publications [3, 6, 7, 8, 9, 10, 11, 25, 26, 28, 29, 30, the authors have used some properties of underlying PDE operators instead of least squares functionals. A very important feature of our numerical method is that it does not require any knowledge of neither the medium inside of the domain of interest nor of any point in a small neighborhood of the true solution. In all these publications convergence analysis was confirmed by numerical examples. Both computationally simulated and experimental data were considered. In particular, the most challenging case of blind real data (i.e. when the solution is unknown in advance) was successfully handled in [25, 29, 30] as well as in chapter 5 and section 6.9 of the book [6].

For the first time, the following two goals were simultaneously achieved for MCIPs for a hyperbolic PDE with single measurement data:

Goal 1. The development of such a numerical method, which would have a rigorous guarantee of obtaining at least one point in a small neighborhood of the exact solution without any advanced knowledge of that neighborhood.

\footnotetext{
* Department of Mathematical Sciences, Chalmers University of Technology and Gothenburg University, SE-42196 Gothenburg, Sweden, (larisa@chalmers.se).

$\dagger$ Department of Mathematics and Statistics, University of North Carolina at Charlotte, Charlotte, NC 28223, USA, (mklibanv@uncc.edu).
} 
Goal 2. This numerical method should have a good performance on computationally simulated data. In addition, if experimental data are available, then this method should also demonstrate a good performance on these data.

It is important to achieve both these goals simultaneously rather than just only one of them. Because of the above mentioned difficulties, one inevitably faces a tough dilemma in an attempt to achieve both Goals 1 and 2: either (1) ignore these goals, or (2) still try to achieve both of them. Because of this dilemma, it is natural to have the rigorous guarantee of Goal 1 within the framework of a reasonable approximate mathematical model (see subsection 3.5). Since convergence is guaranteed in the framework of that model, then we call our numerical method approximately globally convergent. This model is verified via a six-step procedure described in section 2 .

We are unaware about other numerical methods for MCIPs which would: (a) simultaneously achieve Goals 1 and 2 and, at the same time, (b) would not rely on some reasonable approximations, which cannot be rigorously justified.

Compared with [3, 7, 8, 9, 10, 11, 25, 26, 28, 29, 30, there are three main elements of this paper: (1) We propose a new and more convenient than before approximate mathematical model, (2) This model leads to a new convergence analysis, and (3) We test this model on computationally simulated backscattering data, both in $2 \mathrm{~d}$ and $3 \mathrm{~d}$. We point out that we use the approximation of our model only on the first iteration of our method, see the first Remark 3.2 in subsection 3.5.

In the majority of the above cited publications we have considered the case when the data are given at the entire boundary, i.e. the case of complete data collection. In the analytical study of this paper we also work with the case of complete data collection. However, in our numerical studies of section 6 we assume that only the backscattering Dirichlet data are given. Next, we use a numerical observation to assign the Dirichlet boundary condition at the rest of the boundary. Although this condition is an approximate one, numerical results show a good performance.

The case of the backscattering data was also considered in [28] and in sections 6.1-6.7 of the book [6]. The 1-d case of blind experimental backscattering data was considered in [29, 30] and in section 6.9 of the book 6]. However, in all references cited in this paragraph both Dirichlet and Neumann boundary conditions were known at the backscattering part of the boundary. This led to the Quasi-Reversibility Method.

In our first publications about this method the so-called "tail functions" (subsection 3.2) were not estimated [7, 8]. Unlike this, in the new approximate model of the current paper tail functions are estimated on each iteration. Let $C^{k+\alpha}$ be Hölder spaces, where $k \geq 0$ is an integer and $\alpha \in(0,1)$. Estimating $C^{k+\alpha}$ norms of tail functions requires all results of section 4 , and this is the most difficult part of our convergence analysis. Indeed, we estimate functions associated with the fundamental solution of a certain elliptic PDE, which is valid in the entire space $\mathbb{R}^{3}$. However, the classical theory provides such estimates only in bounded domains 31. Although lemmata of section 4 and the main theorems 4.2 and 5.1 were published in the book [6], we believe that it is worthy to publish their complete proofs here as well. This is because journal publications are often better available and for wider audiences of readers than books.

We search for the spatially distributed dielectric constant. We refer to [36] for a different numerical method for an MCIP of calculating the dielectric constant. Another non-local numerical method for a 2-d MCIP for a hyperbolic PDE was developed in [19, 20. It is based on a 2-d analog of the Gel'fand-Levitan-Krein equation. A different version of our approximately globally convergent numerical method was developed in parallel with the above publications for the case of a 2d MCIP for an elliptic PDE with the running source, see [27] and references cited there. The asymptotic behavior of the tail function in this case is radically different from ours, which led to 
a different approximation of tail functions. This problem has an application in medical optical imaging of brains, see, e.g. 37 for imaging from an experimental data set for a phantom medium. A theory of a non-local reconstruction technique for an MCIP for an elliptic PDE with the data given in the form of the scattering amplitude was developed in [32, 33]. We refer to [2] for a numerical implementation of this theory. Note that in numerical studies of non-local reconstruction techniques in [2, 19, 20] some reasonable approximations were used, which cannot be rigorously justified. This is similar with our approximate global convergence concept.

In section 2 we present the notion of the approximate global convergence. In section 3 we describe our algorithm. In particular, we present our approximate mathematical model in subsection 3.5. In section 4 we prove some estimates for the function which is the Laplace transform of the solution of the originating hyperbolic PDE. These estimates are used in section 5 then, where we prove the approximate global convergence theorem 5.1, which is the central analytical result of this paper. In section 6 we present results of our numerical experiments. Summary is given in section 7 .

2. Approximate Global Convergence. To verify our approximate mathematical models, we use the six step procedure:

Step 1. A reasonable approximate mathematical model is proposed. The accuracy of this model cannot be rigorously estimated.

Step 2. A numerical method is developed, which works within the framework of this model.

Step 3. A theorem is proven, which guarantees that, within the framework of this model, the numerical method of Step 2 indeed delivers a point in a sufficiently small neighborhood of the exact solution, provided that the following natural condition is in place: the error, both in the data and in some "secondary" additional approximations, is sufficiently small.

Step 4. The numerical method of Step 2 is tested on computationally simulated data.

Step 5 (optional). The numerical method of Step 2 is tested on experimental data. To have a truly unbiased case, blind data are preferable. This step is optional because it is usually not easy to actually get experimental data.

Step 6. Finally, if results of Step 4 and (optionally) Step 5 are good ones, then Goals 1,2 are simultaneously achieved, and that approximate mathematical model is proclaimed as a valid one.

It is sufficient to achieve that small neighborhood of the exact solution after a finite (rather than infinite) number of iterations. Next, because of approximations in the mathematical model, the resulting solution can be refined via a locally convergent numerical method. We have chosen the Adaptive Finite Element Method (adaptivity) for the latter, see [8, 9, 10, 11] and chapter 4 of 6]. The algorithms of our previous publications were successfully verified on two types of blind experimental data, see [6, 25, 29, 30]. However, since the authors do not posses a proper experimental data for the algorithm of this paper, it is verified here only on computationally simulated data.

The common perception of the term "global convergence" is that one can choose almost any point as the starting point for iterations, and still the process would converge to the correct solution. Actually, however, it is sufficient to start from such a reasonable point, which would not contain any information about a small neighborhood of the exact solution. In addition, it is not necessary to converge to the solution. In fact, it would be sufficient to reach at least one this is going along well with the theory of Ill-Posed problems, see, e.g. Theorem 4.6 of [4] and pages 156, 157 of [17]. Therefore, we come up with Definition 2.1.

Consider a nonlinear ill-posed problem $P$. Suppose that this problem has a unique solution $x^{*} \in B$ for the noiseless data $y^{*}$, where $B$ is a Banach space with the norm $\|\cdot\|_{B} \cdot$ We call $x^{*}$ "exact solution" or "correct solution". Suppose that a certain approximate mathematical model $M$ 
is proposed to solve the problem $P$ numerically. Assume that, within the framework of the model $M$, this problem has unique exact solution $x_{M}^{*}$ and let $x_{M}^{*}=x^{*}$.

Definition 2.1 (approximate global convergence). Consider an iterative numerical method for solving the problem $P$. Suppose that this method produces a sequence of points $\left\{x_{n}\right\}_{n=1}^{N} \subset B$, where the integer $N \in[1, \infty)$. Furthermore, assume that this sequence is produced without any a priori knowledge of a sufficiently small neighborhood of $x^{*}$. Let a sufficiently small number $\varepsilon \in(0,1)$. We call this numerical method approximately globally convergent of the level $\varepsilon$, or shortly globally convergent, if, within the framework of the approximate model $M$, a theorem is proven, which guarantees that there exists numbers $N_{1}, N_{2} \in[1, N], N_{1}<N_{2}$ such that

$$
\left\|x_{n}-x^{*}\right\|_{B} \leq \varepsilon, n \in\left[N_{1}, N_{2}\right]
$$

Suppose that iterations are stopped at a certain number $k \in\left[N_{1}, N_{2}\right]$. Then the point $x_{k}$ is denoted as $x_{k}:=x_{g l o b}$ and is called "the approximate solution resulting from this method".

The validity of the approximate mathematical model $M$ of Definition 2.1 should be verified via above Steps 4,5 . Note that the assumption of the existence of the exact $x^{*}$ for the noiseless data $y^{*}$ is one of the key principles of the theory of Ill-Posed problems [6, 39].

3. The Approximately Globally Convergent Method. This method was described in our above cited publications. However, since we need to prove a new convergence theorem here, then we need to use some formulas of this method in the proof. Hence, we outline it here while still omitting many details for brevity.

3.1. Statements of forward and inverse problems. Let $\Omega \subset \mathbb{R}^{3}$ be a convex bounded domain with the boundary $\partial \Omega \in C^{3}$. Denote $|f|_{k+\alpha}=\|f\|_{C^{k+\alpha}(\bar{\Omega})}, \forall f \in C^{k+\alpha}(\bar{\Omega})$. Let $d=$ const. $>2$. We assume that the coefficient $c(x)$ satisfies the following conditions

$$
c(x) \in[1, d], \quad c(x)=1 \text { for } x \in \mathbb{R}^{3} \backslash \Omega, c \in C^{\alpha}\left(\mathbb{R}^{3}\right) .
$$

We assume a priori knowledge of the constant $d$, which amounts to the knowledge of the correctness set in the theory of Ill-Posed problems [4, 6, 17, 39]. However, we do not assume that the number $d-1$ is small, i.e. we do not impose smallness assumptions on the unknown coefficient $c(x)$. Consider the Cauchy problem for the hyperbolic equation

$$
\begin{aligned}
c(x) u_{t t} & =\Delta u \text { in } \mathbb{R}^{3} \times(0, \infty), \\
u(x, 0) & =0, u_{t}(x, 0)=\delta\left(x-x_{0}\right) .
\end{aligned}
$$

Equation (3.2) governs, e.g. propagation of acoustic and electromagnetic waves. In the acoustical case $c(x)=b^{-2}(x)$, where $b(x)$ is the sound speed. In the 2-D case of EM waves propagation, the dimensionless coefficient is $c(x)=\varepsilon_{r}(x)$, where $\varepsilon_{r}(x)$ is the spatially distributed dielectric constant of the medium. In the latter case the assumption $c(x)=1$ for $x \in \mathbb{R}^{3} \backslash \Omega$ in (3.1) means that we have air outside the medium of interest $\Omega$. And the assumption $c(x) \geq 1$ reflects the fact that the dielectric constants of almost all materials exceed the one of the air. Equation (3.2) was successfully used in [6, 10, 25 to work with experimental data, which are obviously in 3-d. The latter was recently explained in [12, where the Maxwell's system was solved in time domain. It was shown in Test 4 of [12] that the component of the electric field $E(x, t)=\left(E_{1}, E_{2}, E_{3}\right)(x, t)$, which was originally initialized, strongly dominates two other components. 
We now formulate the CIP for the case when the data are given at the entire boundary $\partial \Omega$ of the domain $\Omega$. We show in section 6 how we reduce the problem with backscattering data to this one.

Coefficient Inverse Problem (CIP). Assume that the coefficient $c(x)$ of equation (3.2) satisfies condition (3.1) and is unknown in the domain $\Omega$. Determine the function $c(x)$ for $x \in \Omega$, assuming that the following function $g(x, t)$ is known for a single source position $x_{0} \notin \bar{\Omega}$

$$
u(x, t)=g(x, t), \forall(x, t) \in \partial \Omega \times(0, \infty) .
$$

The function $g(x, t)$ models time dependent measurements of the wave field at the boundary of the domain of interest. Practical measurements are calculated at a number of detectors, of course. In this case the function $g(x, t)$ can be obtained via one of standard interpolation procedures. The assumption of the infinite time interval in (3.4) is not a restrictive one, because we work with the Laplace transform of the function $u(x, t)$ and the kernel of this transform decays rapidly as $t \rightarrow \infty$. Hence, the integral over the interval $(T, \infty)$ is actually discounted in practical computations. Thus, when generating the data for our CIP, we compute the forward problem for $t \in(0, T)$, where $T>0$ is a finite number. Another argument here is that in our work with experimental data [6, 10, 25, 29, 30, we have actually used only a small portion of these data after the data pre-processing procedure.

Global uniqueness theorems for MCIPs with single measurement data are currently known only under the assumption that at least one of initial conditions does not equal zero in the entire domain $\bar{\Omega}$, which is not our case. All these theorems were proven by the method, which was proposed in 1981 by Bukhgeim and Klibanov in [13, 14, 21]; also see, e.g. [15, 22, 23] for some follow up publications of these authors and references in books [6, 24] for publications of many other researchers about this method. This method is based on Carleman estimates. Actually the idea of our approximately globally convergent method of working with an integral differential equation, which does not contain the unknown coefficient $c(x)$, has roots in the method of [13, 14, 15, 21, 22, 23, 24]. There are also some uniqueness theorems for MCIPs with single measurement data for the case when the unknown coefficient has the form const. $+a(x)$, where $\|a\|<<1$, where $\|\cdot\|$ is a certain norm, see, e.g. [35. Our theory below does not rely on any smallness assumptions imposed on $c(x)$. Although we image inclusions of small geometrical sizes in computations, the inclusions/background contrasts are not small. Thus, we have no choice but to assume everywhere below that uniqueness theorem is valid for our CIP.

3.2. Integral differential equation. Consider the Laplace transform of the function $u$,

$$
w(x, s)=\int_{0}^{\infty} u(x, t) e^{-s t} d t, \text { for } s>\underline{s}=\text { const. }>0 .
$$

We assume that the number $\underline{s}$ is sufficiently large, so that the integral (3.5) converges absolutely and the same is valid for the derivatives $D^{k} u, k=0,1,2$. We call the parameter $s$ pseudo frequency. It follows from (3.2), (3.3) and (3.5) that the function $w$ is the solution of the following problem

$$
\begin{aligned}
\Delta w-s^{2} c(x) w & =-\delta\left(x-x_{0}\right), x \in \mathbb{R}^{3}, \\
\lim _{|x| \rightarrow \infty} w(x, s) & =0
\end{aligned}
$$

see Theorem 4.1 below about (3.7). Since $x_{0} \notin \bar{\Omega}$, then Theorem 4.1 also implies that the function $w \in C^{2+\alpha}(\bar{\Omega})$. Suppose that geodesic lines generated by the function $c(x)$ are regular and $c(x)$ 
is sufficiently smooth. Let $\tau\left(x, x_{0}\right)$ be the length of the geodesic line connecting points $x$ and $x_{0}$. Then Theorem 4.1 of 35 implies that the following asymptotic behavior of the function $w(x, s)$ at $s \rightarrow \infty$ takes place [7, 6]

$$
\left|D_{s}^{k} w(x, s)\right|_{2+\alpha}=\left|D_{s}^{k}\left\{\frac{\exp \left[-s \tau\left(x, x_{0}\right)\right]}{f\left(x, x_{0}\right)}\right\}\right|_{2+\alpha}\left[1+O\left(\frac{1}{s}\right)\right], s \rightarrow \infty, k=0,1,
$$

where $f\left(x, x_{0}\right)$ is a certain function and $f\left(x, x_{0}\right) \neq 0$ for $x \in \bar{\Omega}$. It is unclear how to effectively verify the regularity of geodesic lines for generic functions $c(x)$. Therefore, we assume below the asymptotic behavior (3.8) without linking it to the regularity of geodesic lines. We verify the asymptotics (3.8) computationally, see [7] and page 173 of [6].

It follows from Theorem 4.1 (below) that the function $w(x, s)>0$. Denote

$$
v(x, s):=\frac{\ln w(x, s)}{s^{2}}
$$

Assuming that (3.8) holds, we obtain

$$
|v(x, s)|_{2+\alpha}=O\left(s^{-k-1}\right), s \rightarrow \infty, k=0,1 .
$$

Keeping in mind that the source $x_{0} \notin \bar{\Omega}$, we obtain

$$
\Delta v+s^{2}(\nabla v)^{2}=c(x), x \in \Omega .
$$

Differentiate both sides of (3.11) with respect to $s$ and let $q(x, s)=\partial_{s} v(x, s)$. Hence,

$$
\begin{aligned}
& v(x, s)=-\int_{s}^{\bar{s}} q(x, \tau) d \tau+V(x, \bar{s}), \\
& V(x, \bar{s})=v(x, \bar{s})=\frac{\ln w(x, \bar{s})}{\bar{s}^{2}} .
\end{aligned}
$$

Here the truncation pseudo frequency $\bar{s}>\underline{s}$ is a large number. We call $V(x, \bar{s})$ the "tail function", and this function is unknown. By (3.10)

$$
|V(x, \bar{s})|_{2+\alpha}=O\left(\bar{s}^{-1}\right), \quad\left|\partial_{\bar{s}} V(x, \bar{s})\right|_{2+\alpha}=O\left(\bar{s}^{-2}\right), \bar{s} \rightarrow \infty \text {. }
$$

The number $\bar{s}$ is the main regularization parameter of our numerical method. In the computational practice $\bar{s}$ is chosen in numerical experiments.

Thus, we obtain from (3.11), (3.12) the following nonlinear integral differential equation

$$
\begin{aligned}
& \Delta q-2 s^{2} \nabla q \int_{s}^{\bar{s}} \nabla q(x, \tau) d \tau+2 s\left[\int_{s}^{\bar{s}} \nabla q(x, \tau) d \tau\right]^{2} \\
& +2 s^{2} \nabla q \nabla V-4 s \nabla V \int_{s}^{\bar{s}} \nabla q(x, \tau) d \tau+2 s(\nabla V)^{2}=0, x \in \Omega .
\end{aligned}
$$


By (3.4) the following Dirichlet boundary condition is given for the function $q$

$$
q(x, s)=\psi(x, s), \forall(x, s) \in \partial \Omega \times[\underline{s}, \bar{s}],
$$

where $\psi(x, s)=s^{-2} \partial_{s} \ln \varphi-2 s^{-3} \ln \varphi$ and $\varphi(x, s)$ is the Laplace transform (3.5) of the function $g(x, t)$ in (3.4). Equation (3.15) has two unknown functions $q$ and $V$. Therefore, to approximate both these functions, we approximate the function $q$ in "inner" iterations and the function $V$ is approximated in "outer" iterations, see Remark 3.1 in subsection 3.4.

Suppose for a moment that functions $q$ and $V$ are approximated in $\Omega$ together with their derivatives $D_{x}^{\beta} q, D_{x}^{\beta} V,|\beta| \leq 2$. Then the corresponding approximation for the target coefficient can be found via (3.11) as

$$
c(x)=\Delta v+\underline{s}^{2}(\nabla v)^{2}, x \in \Omega,
$$

where the function $v$ is approximated via (3.12). Although any value of the pseudo frequency $s \in[\underline{s}, \bar{s}]$ can be used in (3.17), we have found in our numerical experiments that the best value is $s:=\underline{s}$.

3.3. Discretization with respect to $s$. We assume that $q(x, s)$ is a piecewise constant function with respect $s$. Hence, we assume that there exists a partition

$$
\underline{s}=s_{N}<s_{N-1}<\ldots<s_{1}<s_{0}=\bar{s}, s_{i-1}-s_{i}=h
$$

of the interval $[\underline{s}, \bar{s}]$ with a sufficiently small grid step size $h$ such that

$$
q(x, s)=q_{n}(x) \text { for } s \in\left(s_{n}, s_{n-1}\right], q_{0} \equiv 0 .
$$

We approximate the boundary condition (3.10) as a piecewise constant function, $q_{n}(x)=\bar{\psi}_{n}(x), x \in$ $\partial \Omega$, where $\bar{\psi}_{n}(x)$ is the average of the function $\psi(x, s)$ over the interval $\left(s_{n}, s_{n-1}\right)$. Next, a certain system of elliptic equations for functions $q_{n}(x)$ is derived from (3.15) using the $s$-dependent so-called "Carleman Weight Function" $\exp \left[\lambda\left(s-s_{n-1}\right)\right], s \in\left(s_{n}, s_{n-1}\right)$, where $\lambda>>1$ is a certain parameter of ones choice. Certain numbers $A_{1, n}, A_{2, n}, I_{1, n}, I_{0}$, which can be analytically calculated, are involved in that system, and the following estimates hold

$$
\begin{aligned}
\left|A_{1, n}\right|+\left|A_{2, n}\right| & \leq 8 \bar{s}^{2}, \\
\left|\frac{I_{1, n}}{I_{0}}\right| & \leq \frac{4 \bar{s}^{2}}{\lambda}, \text { if } \lambda h \geq 1,
\end{aligned}
$$

Because of (3.21) we choose in our computations the parameter $\lambda>>1$ so large that we can ignore the nonlinear term $\left(\nabla q_{n}\right)^{2}$ in that system. Thus, we set below

$$
2 \frac{I_{1, n}}{I_{0}}\left(\nabla q_{n}\right)^{2}:=0 .
$$

Our algorithm reconstructs iterative approximations $c_{n, i}(x) \in C^{\alpha}(\bar{\Omega})$ of the function $c(x)$ only inside the domain $\Omega$. To work with our algorithm, we should extend each function $c_{n, i}(x)$ outside of the domain $\Omega$. To do this, choose a smaller subdomain $\Omega^{\prime} \subset \Omega, \partial \Omega^{\prime} \cap \partial \Omega=\varnothing$. Let the function $\chi(x)$ be such that

$$
\chi \in C^{1}\left(\mathbb{R}^{3}\right), \chi(x)=\left\{\begin{array}{c}
1 \text { in } \Omega^{\prime} \\
\in[0,1] \text { in } \Omega \backslash \Omega^{\prime} \\
0 \text { outside of } \Omega .
\end{array}\right.
$$


The existence of such functions $\chi(x)$ is well known from the Real Analysis course. Let the number $l \geq d$. Consider the set of functions $Q(d, l) \subset C^{\alpha}(\bar{\Omega})$ defined as

$$
Q(d, l)=\left\{c \in C^{\alpha}(\bar{\Omega}): c \in[1, d],|c|_{\alpha} \leq l\right\} .
$$

We assume in our algorithm that all functions $c_{n, i} \in Q(d, l)$. Consider the function $\bar{c}_{n, i}(x)$,

$$
\bar{c}_{n, i}(x):=(1-\chi(x))+\chi(x) c_{n, i}(x), \forall x \in \mathbb{R}^{3} .
$$

Then (3.23) and (3.24) imply that

$$
\bar{c}_{n, i} \in[1, d], \bar{c}_{n, i} \in C^{\alpha}\left(\mathbb{R}^{3}\right), \bar{c}(x)=1 \text { for } x \in \mathbb{R}^{3} \backslash \Omega .
$$

3.4. The Algorithm. We now describe our algorithm for approximating functions $q_{n}$ and $V$. Following (3.12), (3.17) and (3.19), denote

$$
\begin{aligned}
& v_{n, i}(x)=-h q_{n, i}(x)-h \sum_{j=0}^{n-1} q_{j}(x)+V_{n, i}(x), x \in \Omega, \\
& c_{n, i}(x)=\left[\Delta v_{n, i}+s_{n}^{2}\left(\nabla v_{n, i}\right)^{2}\right](x), x \in \Omega, i=1, \ldots, m,
\end{aligned}
$$

where functions $q_{j}, q_{n, i}, V_{n, i}$ are defined in this subsection below and $m$ is the number of iterations with respect to tails for each given $n \geq 1$. The number $m$ is chosen in numerical experiments. Here $V_{n, i}(x)$ is a certain approximation for the tail function. Let $V_{1,1}(x)$ be the first guess for the tail function, which is described in subsection 3.5. Hence, to start our iterative process, we set

$$
q_{0}:=0 \text {. }
$$

Step $n_{i}, i \in[1, m], n \geq 1$, see (3.27) for $q_{0}$. For each $n$ we iterate with respect to the tails. Suppose that functions $q_{j}, V_{n, i}(x, \bar{s}) \in C^{2+\alpha}(\bar{\Omega}), j \in[0, n-1]$ are constructed. Then we solve the following Dirichlet boundary value problem for the function $q_{n, i}$

$$
\begin{aligned}
& \Delta q_{n, i}-A_{1 n}\left(h \sum_{j=0}^{n-1} \nabla q_{j}\right) \cdot \nabla q_{n, i}+A_{1 n} \nabla q_{n, i} \cdot \nabla V_{n, i}= \\
& -A_{2 n} h^{2}\left(\sum_{j=0}^{n-1} \nabla q_{j}\right)^{2}+2 A_{2 n} \nabla V_{n, i} \cdot\left(h \sum_{j=0}^{n-1} \nabla q_{j}\right)-A_{2 n}\left(\nabla V_{n, i}\right)^{2}, x \in \Omega, \\
& \left.q_{n, i}\right|_{\partial \Omega}=\bar{\psi}_{n}(x),
\end{aligned}
$$

Because of (3.22), the nonlinear term with $\left(\nabla q_{n, i}\right)^{2}$ is ignored in (3.28). Having the function $q_{n, i}$, we reconstruct the next approximation $c_{n, i} \in C^{\alpha}(\bar{\Omega})$ for the target coefficient using (3.25), (3.26). Next, we construct the function $\bar{c}_{n, i} \in C^{\alpha}\left(\mathbb{R}^{3}\right)$ via (3.24). Next, we calculate the solution $u_{n, i}(x, t)$ of the forward problem (3.2), (3.3) with $c(x):=\bar{c}_{n, i}(x)$. Next, we calculate the Laplace transform $w_{n, i}(x, \bar{s})$ (3.5) of the function $u_{n, i}(x, t)$ at $s:=\bar{s}$ and update the tail function using (3.13),

$$
\frac{\ln w_{n, i}(x, \bar{s})}{\bar{s}^{2}}=\left\{\begin{array}{c}
V_{n, i+1}(x) \text { if } i \in[1, m-1] \\
V_{n+1,1}(x) \text { if } i=m \text { and } n \in[1, N-1]
\end{array}\right.
$$


We set

$$
q_{n}:=q_{n, m} \in C^{2+\alpha}(\bar{\Omega}), c_{n}:=c_{n, m} \in C^{\alpha}(\bar{\Omega}) .
$$

If $i=m$ and $n=N$, then we stop. In fact, we can stop the iterative process not only at $n:=N$ but at $n:=\bar{N} \in[1, N)$ as well. The stopping rule is chosen in numerical experiments, see section 6.

Remark 3.1. It is clear from (3.28)-(3.30) that functions $q_{n, i}$ are updated via inner iterations while "being inside" the domain $\Omega$ only. But to update tail functions $V_{n, i}$, we "go outside of $\Omega$ ", thus, using outer iterations.

3.5. The new approximate mathematical model and the first guess $V_{1,1}(x)$ for the tail. Following the Tikhonov concept [6, 39], we assume that there exists unique exact solution $c^{*}(x)$ of our CIP with noiseless data $g^{*}(x, t)$ (3.4). We assume that

$$
c^{*} \in C^{\alpha}\left(\mathbb{R}^{3}\right), c^{*}(x) \in[1, d-1] \text { in } \mathbb{R}^{3}, c^{*}(x)=1 \text { for } x \in \mathbb{R}^{3} \backslash \Omega^{\prime},\left|c^{*}\right|_{\alpha} \leq l-1 .
$$

For each function $c \in Q(d, l)$ denote $w_{\bar{c}}(x, s)$ the unique solution of the problem (3.6), (3.7) with $c:=\bar{c}$ satisfying conditions (4.2)-(4.5) (Theorem 4.1), where the function $\bar{c}$ is defined in (3.24). Let $w^{*}(x, s)$ be the solution of the problem (3.6), (3.7) with $c:=c^{*}$ satisfying conditions (4.2), (4.3). Then (4.4) and (4.5) are also valid for $w^{*}(x, s)$ (see Theorem 4.1 in subsection 4.1). Using (3.13), we define tails $V_{\bar{c}}(x, s), V^{*}(x, s)$ as

$$
V_{\bar{c}}(x, s)=\frac{\ln w_{\bar{c}}(x, s)}{s^{2}}, V^{*}(x, s)=\frac{\ln w^{*}(x, s)}{s^{2}}, \forall s \geq \bar{s} .
$$

We call $V^{*}(x, s)$ the "exact tail". Assuming that the asymptotic behavior (3.8) holds, we obtain

$$
V^{*}(x, s)=\frac{p^{*}(x)}{s}+O\left(\frac{1}{s^{2}}\right), s \rightarrow \infty, x \in \bar{\Omega} .
$$

for a certain function $p^{*}(x)$. We truncate the second term of this asymptotic behavior. Thus, our new approximate mathematical model consists of the following assumption.

Assumption. There exists a function $p^{*}(x) \in C^{2+\alpha}(\bar{\Omega})$ such that the exact tail function $V^{*}(x, s)$ has the form

$$
V^{*}(x, s):=\frac{p^{*}(x)}{s} . \text { Furthermore, } \frac{p^{*}(x)}{s}=\frac{\ln w^{*}(x, s)}{s^{2}}, \forall s \geq \bar{s} .
$$

Since $q^{*}(x, s)=\partial_{s} V^{*}(x, s)$ for $s \geq \bar{s}$, we derive from (3.34) that

$$
q^{*}(x, \bar{s})=-\frac{p^{*}(x)}{\bar{s}^{2}} .
$$

Set in (3.15) $s=\bar{s}$. Then, using (3.34) and (3.35), we obtain the following approximate Dirichlet boundary value problem for the function $p^{*}(x)$

$$
\begin{aligned}
\Delta p^{*} & =0 \text { in } \Omega, p^{*} \in C^{2+\alpha}(\bar{\Omega}), \\
\left.p^{*}\right|_{\partial \Omega} & =-\bar{s}^{2} \psi^{*}(x, \bar{s}),
\end{aligned}
$$


where $\psi^{*}(x, s)$ is the exact function $\psi(x, s)$, which corresponds to the function $g^{*}(x, t)$. The approximate equation (3.36) is valid only within the framework of the above Assumption. Although this equation is linear, formula (3.17) for the reconstruction of the target coefficient $c^{*}$ is nonlinear.

Recall that by (3.16) $q(x, s)=\psi(x, s), \forall(x, s) \in \partial \Omega \times[\underline{s}, \bar{s}]$. Assume that

$$
\psi(x, s) \in C^{2+\alpha}(\bar{\Omega}), \forall s \in[\underline{s}, \bar{s}] .
$$

Consider the solution $p(x)$ of the following boundary value problem

$$
\begin{aligned}
\Delta p & =0 \text { in } \Omega, p \in C^{2+\alpha}(\bar{\Omega}), \\
\left.p\right|_{\partial \Omega} & =-\bar{s}^{2} \psi(x, \bar{s}) .
\end{aligned}
$$

As the first guess for the tail function we take

$$
V_{1,1}(x):=\frac{p(x)}{\bar{s}} .
$$

By the Schauder theorem there exists unique solution $p$ of the problem (3.39), (3.40). Furthermore, it follows from (3.36)-(3.41) and Schauder theorem that with a number $M=M(\Omega)>0$ the following estimates hold

$$
\begin{aligned}
\left|\nabla V_{1,1}-\nabla V^{*}\right|_{1+\alpha} & \leq M\left\|\psi(x, \bar{s})-\psi^{*}(x, \bar{s})\right\|_{C^{2+\alpha}(\partial \Omega)} \\
\left|\nabla V_{1,1}\right|_{1+\alpha} & \leq M \bar{s}\|\psi(x, \bar{s})\|_{C^{2+\alpha}(\partial \Omega)} .
\end{aligned}
$$

Remarks 3.2.

1. The main approximation is the second equality (3.34). This approximation amounts to the truncation of the second term $O\left(s^{-2}\right)$ of the asymptotics (3.33). It is made only to obtain the estimate (3.42) on the first iteration of our method to obtain estimate (5.36) in the proof of the convergence Theorem 5.1. On all follow up iterations in that proof we do not use the second equality (3.34). Rather, we use the true fact that $V^{*}(x)=\bar{s}^{-2} \ln w^{*}(x, \bar{s})$.

2. It follows from (3.42) that, substituting (3.41) in (3.25) and (3.26) at $n=1$ and setting $q_{1, i}:=0$, we obtain a good approximation for the exact solution already on the first iteration of our method, as long as the error in the boundary data $\psi^{*}(x, \bar{s})$ is small. The smallness of the error is a natural assumption. Theorem 5.1 guarantees that all other solutions obtained in the iterative process of subsection 3.4 also provide good approximations, as long as the number of iterations is not too large. This means that we should develop numerically a stopping criterion to stop iterations, see section 6. Suppose now that iterations are stopped before this stopping criterion is met, e.g. just on the first iteration. In this case we can apply the second stage of our two-stage numerical procedure [6, 8, 9, 10, 11. Namely, we could apply a locally convergent numerical method to refine the solution via taking the solution obtained on the globally convergent stage as the starting point of iterations. Such a method can be applied indeed, since Theorem 5.1 guarantees that the iterative solution, at which we have stopped, is close to the exact solution $c^{*}(x)$. Numerical confirmations of this can be found in tests 2,3 of [8] and in tests 2,3 of section 4.16 .2 of [6].

We now establish uniqueness within the framework of our approximate mathematical model. We refer to Lemma 2.9.2 of [6] for the proof of Lemma 3.1.

Lemma 3.1. Let the above Assumption holds. In addition, let (3.17) holds for the function $v^{*}(x, s)$,

$$
v^{*}(x, s)=-\int_{s}^{\bar{s}} q^{*}(x, \tau) d \tau+V^{*}(x, \bar{s})
$$


with the tail function $V^{*}(x, s)$ satisfying conditions (3.34), (3.36) and (3.37), i.e.

$$
c^{*}(x)=\left[\Delta v^{*}+s^{2}\left|\nabla v^{*}\right|^{2}\right](x, s),(x, s) \in \Omega \times[\underline{s}, \bar{s}] .
$$

Then there exists at most one function $c^{*}(x)$.

\section{Estimates of Tails.}

4.1. Some estimates of the function $w(x, s)$. First, we should justify (3.6), (3.7). Theorem 4.1 is a combination of Theorems 2.7.1 and 2.7.2 of [6]. Therefore we refer to [6] for the proof.

Theorem 4.1. Assume that the coefficient $c(x)$ of equation (3.2) is such that

$$
c \in C^{k+\alpha}\left(\mathbb{R}^{3}\right), c(x) \in[1, d], \quad c(x)=1 \text { for } x \in \mathbb{R}^{3} \backslash \Omega .
$$

Assume that the solution $u(x, t)$ of the problem (3.2), (3.3) is such that

$$
u \in C^{2}\left(t \geq M_{1}\left(x, x_{0}, c\right)\right),\left|D^{\gamma} u(x, t)\right| \leq M_{2}\left(x_{0}, c\right) e^{\beta t},|\gamma| \leq 2, \forall x \in \mathbb{R}^{3}, \forall t \geq M_{1}\left(x, x_{0}, c\right),
$$

where $M_{1}\left(x, x_{0}, c\right)$ and $\beta=\beta(c)$ are positive numbers depending on listed parameters. Let the function $w(x, s)$ be the Laplace transform (3.5) of the function $u(x, t)$. Then there exists a number $\underline{s}=\underline{s}(c)>\beta(c)$ such that for all $s \geq \underline{s}(c)$ the function $w(x, s)$ is the unique solution of the problem (3.6), (3.7) of the form

$$
\begin{aligned}
w(x, s) & =\frac{\exp \left(-s\left|x-x_{0}\right|\right)}{4 \pi\left|x-x_{0}\right|}+\bar{w}(x, s):=w_{1}(x, s)+\bar{w}(x, s), \\
\bar{w} & \in C^{k+2+\alpha}\left(\mathbb{R}^{3}\right) .
\end{aligned}
$$

Also, the following inequalities hold

$$
w_{d}(x, s)<w(x, s) \leq w_{1}(x, s), \forall x \neq x_{0},
$$

where the function $w_{d}(x, s)$ is the unique solution of the problem (3.6), (3.7) for the case $c(x) \equiv d$,

$$
w_{d}(x, s)=\frac{\exp \left(-s \sqrt{d}\left|x-x_{0}\right|\right)}{4 \pi\left|x-x_{0}\right|} .
$$

Furthermore, consider the problem (3.6), (3.7) irrelevantly to the problem (3.2), (3.3) while still assuming (4.1). Then for any $s>0$ there exists unique solution $w(x, s)$ of this problem satisfying conditions (4.2), (4.3). Furthermore, conditions (4.4), (4.5) hold for this function $w(x, s)$.

Lemma 4.1. Let $\chi(x)$ be the function defined in (3.23), functions $c_{1}, c_{2} \in Q(d, l)$ and functions $\bar{c}_{1}(x), \bar{c}_{1}(x)$ be defined as in (3.24). Then $\left|\bar{c}_{1}-\bar{c}_{2}\right|_{\alpha} \leq|\chi|_{\alpha}\left|c_{1}-c_{2}\right|_{\alpha}$.

Proof. By (3.24) $\bar{c}_{1}(x)-\bar{c}_{2}(x)=\chi(x)\left(c_{1}(x)-c_{2}(x)\right)$. The rest of the proof follows from $|f g|_{\alpha} \leq|f|_{\alpha}|g|_{\alpha}, \forall f, g \in C^{\alpha}(\bar{\Omega})$.

Note that $C^{1}(\bar{\Omega}) \subset C^{\alpha}(\bar{\Omega})$, and also there exists a constant $C=C(\Omega, \alpha)>0$ such that

$$
|f|_{\alpha} \leq C\|f\|_{C^{1}(\bar{\Omega})}, \forall f \in C^{1}(\bar{\Omega}) .
$$

Lemma 4.2. Let the source $x_{0} \notin \bar{\Omega}$. Then there exists a constant $Y=Y\left(\Omega, \bar{s}, d, l, \chi, x_{0}, \alpha\right)>0$ depending on listed parameters such that $\left|w_{\bar{c}}(x, \bar{s})\right|_{\alpha} \leq Y, \forall c \in Q(d, l)$. 
Proof. Below in this proof $Y=Y\left(\Omega, \bar{s}, d, l, \chi, x_{0}, \alpha\right)>0$ denotes different constants depending on listed parameters. By (4.2) and (4.3) $w_{\bar{c}}(x, \bar{s}) \in C^{2+\alpha}(\bar{\Omega})$. Denote $b(x)=\bar{c}(x)-1$. Then

$$
w_{\bar{c}}(x, \bar{s})=w_{0}(x, \bar{s})-\bar{s}^{2} \int_{\Omega} w_{1}(x-\xi, \bar{s}) b(\xi) w_{\bar{c}}(\xi, \bar{s}) d \xi .
$$

By (4.4) and (4.7)

$$
\left|w_{\bar{c}}(x, \bar{s})\right| \leq Y+Y\|b\|_{C(\bar{\Omega})} \int_{\Omega} w_{1}(x-\xi, \bar{s}) d \xi \leq Y, x \in \Omega .
$$

In addition, by (4.7)

$$
\nabla w_{\bar{c}}(x, \bar{s})=\nabla w_{0}(x, \bar{s})-\bar{s}^{2} \int_{\Omega} \nabla w_{1}(x-\xi, \bar{s}) b(\xi) w_{\bar{c}}(\xi, \bar{s}) d \xi, x \in \Omega .
$$

Hence, $\left|\nabla w_{\bar{c}}(x, \bar{s})\right| \leq Y, x \in \Omega$. Hence, (4.6), (4.8) and (4.9) imply that $\left|w_{\bar{c}}(x, \bar{s})\right|_{\alpha} \leq Y$.

Consider a bounded domain $\Omega_{1} \subset \mathbb{R}^{3}$ such that

$$
\Omega \subset \Omega_{1}, \partial \Omega \cap \partial \Omega_{1}=\varnothing, \partial \Omega_{1} \in C^{3}, x_{0} \notin \bar{\Omega}_{1} .
$$

Lemma 4.3. Let $\Omega^{\prime} \subset \Omega \subset \Omega_{1}$ be above bounded domains in $\mathbb{R}^{3}$, condition $(\sqrt{4.10})$ be satisfied and $\chi(x)$ be the function in (3.23). Let $\bar{s} \geq 1$. Then the function $w_{\bar{c}}(x, \bar{s}) \in C^{3}\left(\partial \Omega_{1}\right), \forall c \in$ $Q(d, l)$. Furthermore, there exists a constant $B=B\left(\Omega, \Omega^{\prime}, \Omega_{1}, \bar{s}, d, l, \chi, x_{0}, \alpha\right)>2$ depending only on listed parameters such that

$$
\left\|w_{\bar{c}}(x, \bar{s})\right\|_{C^{3}\left(\partial \Omega_{1}\right)} \leq B, \forall c \in Q(d, l) .
$$

For any two functions $c_{1}, c_{2} \in Q(d, l)$ denote $\widetilde{w}(x)=w_{\bar{c}_{1}}(x, \bar{s})-w_{\bar{c}_{2}}(x, \bar{s})$. Then

$$
\|\widetilde{w}\|_{C^{3}\left(\partial \Omega_{1}\right)} \leq B\left|c_{1}-c_{2}\right|_{\alpha}, \forall c_{1}, c_{2} \in Q(d, l) .
$$

Proof. Everywhere below in this paper $B$ denotes different positive constant depending on above parameters. The integrand of formula (4.7) does not have a singularity for $x \in \Omega_{1} \backslash \bar{\Omega}$. Hence, (4.7) implies that $w_{\bar{c}}(x, \bar{s}) \in C^{3}\left(\partial \Omega_{1}\right)$. Next, (4.11) follows from (4.7) and Lemma 4.2.

Denote

$$
\widetilde{c}(x)=c_{1}(x)-c_{2}(x), b_{1}(x)=c_{1}(x)-1, b_{2}(x)=c_{2}(x)-1 .
$$

Hence, by (3.24) $\bar{c}_{1}(x)-\bar{c}_{2}(x)=\chi(x) \widetilde{c}(x)$. First, substitute in (4.7) $\left(b_{1}, w_{\bar{c}_{1}}\right)$. Next, substitute $\left(b_{2}, w_{\bar{c}_{2}}\right)$. Next, subtract the second equation from the first one and denote $\widetilde{w}(x)=w_{\bar{c}_{1}}(x, \bar{s})-$ $w_{\bar{c}_{2}}(x, \bar{s})$. We obtain

$$
\widetilde{w}(x)=-\bar{s}^{2} \int_{\Omega} w_{1}(x-\xi, \bar{s}) \chi(\xi) \widetilde{c}(\xi) w_{\bar{c}_{1}}(\xi, \bar{s}) d \xi-\bar{s}^{2} \int_{\Omega} w_{1}(x-\xi, \bar{s}) b_{2}(\xi) \widetilde{w}(\xi) d \xi .
$$


Let

$$
\begin{aligned}
& I_{1}(x)=-\bar{s}^{2} \int_{\Omega} w_{1}(x-\xi, \bar{s}) \chi(\xi) \widetilde{c}(\xi) w_{\bar{c}_{1}}(\xi, \bar{s}) d \xi \\
& I_{2}(x)=-\bar{s}^{2} \int_{\Omega} w_{1}(x-\xi, \bar{s}) b_{2}(\xi) \widetilde{w}(\xi) d \xi .
\end{aligned}
$$

Using the same arguments as ones in the proof of (4.11), we obtain

$$
\left\|I_{1}\right\|_{C^{3}\left(\partial \Omega_{1}\right)} \leq B|\widetilde{c}|_{\alpha} .
$$

Next,

$$
\Delta \widetilde{w}-\bar{s}^{2} \bar{c}_{2} \widetilde{w}=\bar{s}^{2} \chi(x) \widetilde{c}(x) w_{\bar{c}_{1}}(x, \bar{s}), x \in \mathbb{R}^{3} .
$$

Furthermore, it follows from (4.13) that the function $\widetilde{w}(x)$ decays exponentially together with its derivatives as $|x| \rightarrow \infty$. Hence, multiplying (4.15) by $\widetilde{w}$, integrating over $\mathbb{R}^{3}$ and using Lemma 4.2 and the fact that $\chi(x)=0$ for $x \in \mathbb{R}^{3} \backslash \Omega$, we obtain in a standard manner $\|\widetilde{w}\|_{L_{2}(\Omega)} \leq B\|\widetilde{c}\|_{L_{2}(\Omega)} \leq$ $B|\widetilde{c}|_{\alpha}$. Hence, $\left\|I_{2}\right\|_{C^{3}\left(\partial \Omega_{1}\right)} \leq B|\widetilde{c}|_{\alpha}$. This estimate combined with (4.14) implies (4.12).

4.2. Estimates of tails. Theorem 4.2. Let $\Omega^{\prime} \subset \Omega \subset \Omega_{1} \subset \mathbb{R}^{3}$ be above bounded domains with condition (4.10), and let $\chi(x)$ be the function in (3.23). Also, let $\bar{s} \geq 1$. For each function $c \in Q(d, l)$ consider the function $\bar{c}$ defined in (3.24). Denote

$$
V_{\bar{c}}(x)=\frac{\ln w_{\bar{c}}(x, \bar{s})}{\bar{s}^{2}} .
$$

Then there exists such a constant $B=B\left(\Omega, \Omega^{\prime}, \Omega_{1}, \bar{s}, d, l, \chi, x_{0}, \alpha\right)>2$ depending only on listed parameters such that

$$
\begin{aligned}
\left|\nabla V_{\bar{c}}\right|_{1+\alpha} & \leq B, \forall c \in Q(d, l), \\
\left|\nabla V_{\bar{c}_{1}}-\nabla V_{\bar{c}_{2}}\right|_{1+\alpha} & \leq B\left|c_{1}-c_{2}\right|_{\alpha}, \forall c_{1}, c_{2} \in Q(d, l) .
\end{aligned}
$$

Proof. By (4.16)

$$
\nabla V_{\bar{c}}(x)=\frac{\nabla w_{\bar{c}}(x, \bar{s})}{\bar{s}^{2} w_{\bar{c}}(x, \bar{s})}, \partial_{x_{i}}^{2} V_{\bar{c}}(x)=\frac{\partial_{x_{i}}^{2} w_{\bar{c}}(x, \bar{s})}{\bar{s}^{2} w_{\bar{c}}(x, \bar{s})}-\frac{\left(\partial_{x_{i}} w_{\bar{c}}(x, \bar{s})\right)^{2}}{\bar{s}^{2} w_{\bar{c}}^{2}(x, \bar{s})}, i=1,2,3 .
$$

Since by (4.4) and (4.5)

$$
\frac{4 \pi \exp \left(\bar{s}\left|x-x_{0}\right|\right)}{\bar{s}^{2}}\left|x-x_{0}\right| \leq \frac{1}{\bar{s}^{2} w_{\bar{c}}(x, \bar{s})}<\frac{4 \pi \exp \left(\bar{s} \sqrt{d}\left|x-x_{0}\right|\right)}{\bar{s}^{2}}\left|x-x_{0}\right| \leq B,
$$

then (4.19) implies that in order to prove (4.17) and (4.18), it is sufficient to prove that

$$
\begin{aligned}
\left|w_{\bar{c}}(x, \bar{s})\right|_{2+\alpha} & \leq B, \forall c \in Q(d, l), \\
\left|w_{\bar{c}_{1}}(x, \bar{s})-w_{c_{2}}(x, \bar{s})\right|_{2+\alpha} & \leq B\left|c_{1}-c_{2}\right|_{\alpha} .
\end{aligned}
$$


Denote $f_{\bar{c}}(x)=\left.w_{\bar{c}}(x, \bar{s})\right|_{\partial \Omega_{1}}$. By Lemma 4.3

$$
f_{\bar{c}}(x) \in C^{3}\left(\partial \Omega_{1}\right),\|f\|_{C^{3}\left(\partial \Omega_{1}\right)} \leq B .
$$

Since by (4.10) $x_{0} \notin \bar{\Omega}_{1}$, then (4.2) and (4.3) imply that $w_{\bar{c}}(x, \bar{s}) \in C^{2+\alpha}\left(\bar{\Omega}_{1}\right)$. On the other hand, the function $w_{\bar{c}}(x, \bar{s})$ solves the following Dirichlet boundary value problem in $\Omega_{1}$

$$
\begin{aligned}
\Delta w_{\bar{c}}-\bar{s}^{2} \bar{c}(x) w_{\bar{c}}=0, x \in \Omega_{1}, \\
w_{\bar{c}} \mid \partial \Omega_{1}=f_{\bar{c}}(x) .
\end{aligned}
$$

Since the $C^{2+\alpha}\left(\bar{\Omega}_{1}\right)$ solution of this problem is unique, then Schauder theorem and (4.22) imply that $\left|w_{\bar{c}}(x, \bar{s})\right|_{2+\alpha} \leq B\|f\|_{C^{2+\alpha}\left(\partial \Omega_{1}\right)} \leq B$, which proves (4.20). Denote again $\widetilde{w}(x)=w_{\bar{c}_{1}}(x, \bar{s})-$ $w_{\bar{c}_{2}}(x, \bar{s})$. Then

$$
\begin{array}{r}
\Delta \widetilde{w}-\bar{s}^{2} \bar{c}_{1}(x) \widetilde{w}=\bar{s}^{2}\left(\bar{c}_{1}(x)-\bar{c}_{2}(x)\right) w_{\bar{c}_{2}}, \\
\widetilde{w} \mid \partial \Omega_{1}=f_{\bar{c}_{1}}(x)-f_{c_{2}}(x) .
\end{array}
$$

Hence, Schauder theorem and Lemmata 4.1 and 4.3 imply (4.21).

\section{Approximate Global Convergence of the Algorithm of Subsection 3.4.}

5.1. Exact solution. Recall that we the existence and uniqueness of the exact solution $c^{*}(x)$ of our MCIP satisfying (3.31). The corresponding functions $w^{*}(x, s), V^{*}(x, \bar{s})$ were defined in subsection 3.5. Let $\underline{s}>\underline{s}\left(c^{*}\right)$, where $\underline{s}\left(c^{*}\right)$ is the number of Theorem 4.1. Let $u^{*}(x, t)$ be the solution of the problem (3.2), (3.3) with $c:=c^{*}$. Since one can differentiate infinitely many times with respect to $s$ under the integral sign in (3.5), then

$$
w^{*}(x, s) \in C^{2+\alpha}(\bar{\Omega}) \times C^{2}[\underline{s}, \bar{s}], \text { if } \underline{s}>\underline{s}\left(c^{*}\right) .
$$

Denote

$$
q^{*}(x, s)=\frac{\partial}{\partial s}\left[\frac{\ln \left[w^{*}(x, s)\right]}{s^{2}}\right], \psi^{*}(x, s)=\left.q^{*}(x, s)\right|_{\partial \Omega}, s \in[\underline{s}, \bar{s}] .
$$

Consider functions $q_{n}^{*}(x), \bar{\psi}_{n}^{*}(x)$,

$$
q_{n}^{*}(x)=\frac{1}{h} \int_{s_{n}}^{s_{n-1}} q^{*}(x, s) d s, \bar{\psi}_{n}^{*}(x)=\frac{1}{h} \int_{s_{n}}^{s_{n-1}} \psi^{*}(x, s) d s, q_{0}^{*}(x) \equiv 0 .
$$

Then (5.1) and (5.2) imply that

$$
\left|q^{*}(x, s)-q_{n}^{*}(x)\right|_{2+\alpha} \leq C^{*} h,\left\|\psi^{*}(x, s)-\bar{\psi}_{n}^{*}(x)\right\|_{C^{2+\alpha}(\partial \Omega)} \leq C^{*} h, n \in[1, N], s \in\left[s_{n}, s_{n-1}\right] .
$$

Here the constant $C^{*}=C^{*}\left(\left\|q^{*}\right\|_{C^{2+\alpha}(\bar{\Omega}) \times C^{1}[\underline{s}, \bar{s}]}, \bar{s}\right)>0$ depends only on the $C^{2+\alpha}(\bar{\Omega}) \times C^{1}[\underline{s}, \bar{s}]$ norm of the function $q^{*}(x, s)$. Hence, we can assume that

$$
\max _{1 \leq n \leq N}\left|q_{n}^{*}\right|_{2+\alpha} \leq C^{*}
$$


Without any loss of generality we assume that

$$
C^{*} \geq 1
$$

By the one of concepts of Tikhonov (see, e. g. section 1.4 of [6]) we assume that the constant $C^{*}$ is known a priori. By (5.2)

$$
q_{n}^{*}(x)=\bar{\psi}_{n}^{*}(x), x \in \partial \Omega .
$$

Hence we obtain the following analogue of equation (3.28)

$$
\begin{aligned}
& \Delta q_{n}^{*}-A_{1, n}\left(h \sum_{j=0}^{n-1} \nabla q_{j}^{*}\right) \nabla q_{n}^{*}+A_{1, n} \nabla q_{n}^{*} \nabla V^{*}=-A_{2, n} h^{2}\left(\sum_{j=0}^{n-1} \nabla q_{i}^{*}\right)^{2} \\
& +2 A_{2, n} \nabla V^{*}\left(h \sum_{j=0}^{n-1} \nabla q_{j}^{*}\right)-A_{2, n}\left|\nabla V^{*}\right|^{2}+F_{n}(x, h, \lambda) .
\end{aligned}
$$

Here the function $F_{n}(x, h, \lambda) \in C^{\alpha}(\bar{\Omega})$. The term $2 I_{1, n}\left(\nabla q_{n}^{*}\right)^{2} / I_{0}$ is included in $F_{n}$, i.e., unlike (3.22), we do not ignore this term now, since we work now with the exact solution. Hence, by (3.21)

$$
\max _{\lambda h \geq 1}\left|F_{n}(x, h, \lambda, \bar{s})\right|_{\alpha} \leq C^{*} h, n \in[1, N] .
$$

Let

$$
v_{n}^{*}(x)=-h q_{n}^{*}(x)-h \sum_{j=0}^{n-1} q_{j}^{*}(x)+V^{*}(x), x \in \Omega, n \in[1, N] .
$$

Using (5.4), we obtain similarly with (3.26)

$$
c^{*}(x)=\left[\Delta v_{n}^{*}+s_{n}^{2}\left|\nabla v_{n}^{*}\right|^{2}\right](x)+\bar{F}_{n}(x),
$$

where the error function $\bar{F}_{n}$ is such that

$$
\left|\bar{F}_{n}\right|_{\alpha} \leq C^{*} h .
$$

We also assume that the function $g(x, t)$ in (3.4) is given with an error. This naturally produces an error in functions $\bar{\psi}_{n}$ in (3.28). Let $\sigma>0$ be a small parameter characterizing the level of the error in the data $\psi(x, s)$. Because of (3.38), we assume that in (3.28) functions $\bar{\psi}_{n}(x) \in C^{2+\alpha}(\partial \Omega)$ and

$$
\left\|\bar{\psi}_{n}^{*}(x)-\bar{\psi}_{n}(x)\right\|_{C^{2+\alpha}(\partial \Omega)} \leq C^{*}(\sigma+h) .
$$

In addition, we assume that We now reformulate the estimate of the Schauder theorem for the specific case we need. Consider the Dirichlet boundary value problem

$$
\begin{aligned}
\Delta u+\sum_{j=1}^{3} b_{j}(x) u_{x_{j}}-b_{0}(x) u & =f(x), x \in \Omega, \\
u & \left.\right|_{\partial \Omega}=g(x) \in C^{2+\alpha}(\partial \Omega) .
\end{aligned}
$$


Assume that the following conditions are in place

$$
b_{j}, b_{0}, f \in C^{\alpha}(\bar{\Omega}), b_{0}(x) \geq 0, \max _{j \in[0, n]}\left(\left|b_{j}\right|_{\alpha}\right) \leq P, P=\text { const. }>0 .
$$

Then Schauder theorem [31 claims that there exists unique solution $u \in C^{2+\alpha}(\bar{\Omega})$ of the boundary value problem (5.14), (5.15), and the following estimate holds with a certain constant $K=$ $K(\Omega, P)>2$, depending only on the domain $\Omega$ and the constant $P$

$$
|u|_{2+\alpha} \leq K\left[\|g\|_{C^{2+\alpha}(\partial \Omega)}+|f|_{\alpha}\right] .
$$

5.2. Approximate global convergence theorem. Theorem 5.1. Let $\Omega^{\prime} \subset \Omega \subset \Omega_{1} \subset \mathbb{R}^{3}$ be above bounded domains with condition (4.10), let $\chi(x)$ be the function in (3.23), $\bar{s} \geq 1$ and (3.38) be valid. Let the function $c^{*}(x)$ satisfying conditions (3.31) be the exact solution of the CIP (3.2)-(3.4), where constants $d, l>1$ are given. Also, let condition (5.13) holds, where $\sigma$ is level of the error in the data, in (3.28) functions $\bar{\psi}_{n} \in C^{2+\alpha}(\partial \Omega)$, and the constant $C^{*}=$ $C^{*}\left(\left\|q^{*}\right\|_{C^{2+\alpha}(\bar{\Omega}) \times C^{1}[\underline{s}, \bar{s}]}, \bar{s}\right) \geq 1$ is defined in 5.4)-(5.6)). Consider the algorithm of subsection 3.4 supplied by Assumption of subsection 3.5. Let the first tail function $V_{1,1}(x)$ be calculated via (3.39), (3.40) and 3.41). In addition, assume that all functions $c_{n, i}(x)$ in (3.26) are such that

$$
c_{n, i}(x) \geq 1, x \in \Omega \text {. }
$$

Assume that the parameter $\lambda$ of the Carleman Weight Function is so large that $\lambda h \geq 1$ (see (3.21)). Let (5.13) be valid and also

$$
\left\|\psi(x, \bar{s})-\psi^{*}(x, \bar{s})\right\|_{C^{2+\alpha}(\partial \Omega)} \leq C^{*} \sigma .
$$

Consider the error parameter $\eta$,

$$
\eta=h+\sigma .
$$

Let $B=B\left(\Omega, \Omega_{1}, \bar{s}, d, l, \chi, x_{0}, \alpha\right)>2$ be the constant of Theorem 4.2. Consider the number $B_{1}=$ $B_{1}\left(\Omega, \Omega_{1}, \bar{s}, d, l, C^{*}, \chi, x_{0}, \alpha\right)$,

$$
B_{1}=\max \left(4 B+3 C^{*}, 24 \bar{s}^{2}\right)>24 .
$$

Let

$$
K=K\left(\Omega, \bar{s}^{2} B_{1}\right) \geq B_{1}
$$

be the constant in (5.17). Let the parameter $\eta$ be so small that

$$
\eta \in\left(0, \eta_{0}\right), \eta_{0}=\frac{1}{K N B_{1}^{3 N m}} .
$$

Then

$$
\begin{aligned}
c_{n, i} & \in C^{\alpha}(\bar{\Omega}), \bar{c}_{n, i} \in C^{\alpha}\left(\mathbb{R}^{3}\right), \quad(n, i) \in[1, N] \times[1, m], \\
c_{n, i}, \bar{c}_{n, i}(x) & \in Q(d, l),(n, i) \in[1, N] \times[1, m] .
\end{aligned}
$$


In addition, the following estimates hold for $(n, i) \in[1, N] \times[1, m]$

$$
\begin{aligned}
\left|\nabla V_{n, i}\right|_{1+\alpha} & \leq B_{1}, \\
\left|\nabla V_{n, i}-\nabla V^{*}\right|_{1+\alpha} & \leq B_{1}^{3[i-1+(n-1) m]+1} \cdot \eta, \\
\left|q_{n, i}-q_{n}^{*}\right|_{2+\alpha} & \leq K B_{1}^{3[i+(n-1) m]} \cdot \eta, \\
\left|q_{n, i}\right|_{2+\alpha},\left|q_{n}\right|_{2+\alpha} & \leq 2 C^{*}, n \in[1, N], \\
\left|c_{n, i}-c^{*}\right|_{\alpha} & \leq B_{1}^{3[i+(n-1) m]} \cdot \eta .
\end{aligned}
$$

Define the number $\omega \in(0,1)$ as

$$
\omega=\frac{\ln (K N)}{2\left[3 N m \ln B_{1}+\ln (K N)\right]}, \omega \in(0,1) .
$$

Then (5.30) becomes

$$
\left|c_{n, i}-c^{*}\right|_{\alpha} \leq \eta^{\omega}:=\varepsilon \in(0,1) .
$$

Therefore, by (5.32) and Definition 2.1 the algorithm of subsection 3.4 possesses the approximate globally convergent property of the level $\varepsilon$.

Remarks 5.1:

1. Since $K=K\left(\Omega, \bar{s}^{2} B_{1}\right)$, one can incorporate the term $K N$ in (5.23) in the term $B_{1}^{3 N m}$. However, we are not doing this for the convenience of the proof.

2. Condition (5.23) provides a linkage between the level of the error $\eta$ in the data and the total "allowable" number of iterations $\mathrm{Nm}$. The fact that the maximal number of iterations $\mathrm{Nm}$ is limited is going along well with the theory of Ill-Posed Problems. Indeed, it is well known that the maximal number of iterations and the error in the data are often connected with each other, see, e.g. pages 156 and 157 of [17] and section 1.6 of [6]. Hence, So that the maximal number of iterations $N m$ is a regularization parameter in this case. The fact that the constant $B_{1}$ depends not only on the domain $\Omega$ but also on the domain $\Omega_{1}$ does not affect the approximate global convergence property.

3. It is hard to establish a priori the upper limit for the maximal number of functions $q_{n}$. We have consistently observed in our numerical tests that certain numbers indicating convergence stabilize a few iterations before a certain number $\bar{N} \in[1, N]$, i.e. at a certain $n<\bar{N}$. Next, they grow steeply for $n_{\widetilde{N}} \geq \bar{N}$. This means that the process should be stopped at a certain $n=\widetilde{N}<\bar{N}$. Usually we take $\widetilde{N}:=\bar{N}-1$, see, e.g. pages $178-182$ and 311-314 in the book [6]. This numerical observation is going along well with (5.23), (5.32).

Proof of Theorem 5.1. Estimate (5.32) follows from estimates (5.23), (5.30) and (5.31). Hence, we focus below on the proof of relations (5.24)-(5.30). Denote

$$
\begin{aligned}
& \widetilde{V}_{n, i}=V_{n, i}-V^{*}, \widetilde{q}_{n, i}=q_{n, i}-q_{n}^{*}, \widetilde{q}_{n}=q_{n}-q_{n}^{*}, \\
& \widetilde{v}_{n, i}=v_{n, i}-v_{n, i}^{*}, \widetilde{c}_{n, i}=c_{n, i}-c^{*}, \widetilde{\psi}_{n}=\bar{\psi}_{n}-\bar{\psi}_{n}^{*} .
\end{aligned}
$$

By (3.23), (3.24) and (3.31) $c^{*}(x) \equiv \bar{c}^{*}(x)$. Hence, we can apply Theorem 4.2 to estimate the norm $\left|\nabla \widetilde{V}_{n, i}\right|_{1+\alpha}$. Suppose that estimate (5.30) holds. Then $c_{n, i} \in Q(d, l)$. Indeed, using (3.31), (5.23) and (5.30), we obtain

$$
\left|c_{n, i}\right|_{\alpha}=\left|c_{n, i}-c^{*}+c^{*}\right|_{\alpha} \leq\left|c^{*}\right|_{\alpha}+\left|c_{n, i}-c^{*}\right|_{\alpha} \leq l-1+B_{1}^{3[i+(n-1) m]} \cdot \eta<l .
$$


Similarly $c_{n, i} \leq d$. The latter two estimates and (5.18) imply that $c_{n, i} \in Q(d, l)$. Next, since the function $c_{n, i} \in Q(d, l)$, then, using (4.17) and (5.21), we obtain (5.26). Also, since the function $c_{n, i} \in[1, d]$, then the function $\bar{c}_{n, i} \in[1, d]$. Hence, if (5.30) is true, then (5.24) and (5.25) hold.

First, we prove (5.28)-(5.30) for the case $(n, i)=(1,1)$. Subtracting equation (5.8) from equation (3.28) at $(n, i)=(1,1)$ and also subtracting (5.7) from the boundary condition in (3.28), we obtain

$$
\begin{aligned}
\Delta \widetilde{q}_{1,1}+A_{1,1} \nabla V_{1,1} \nabla \widetilde{q}_{1,1} & =-A_{1,1} \nabla \widetilde{V}_{1,1} \nabla q_{1}^{*}-A_{2,1} \nabla \widetilde{V}_{1,1}\left(\nabla V_{1,1}+\nabla V^{*}\right)-F_{1} \\
\widetilde{q}_{1,1}(x) & =\widetilde{\psi}_{1}(x), x \in \partial \Omega .
\end{aligned}
$$

We now estimate the right hand side of (5.33). It follows from (3.31), (3.32) and (4.17) that

$$
\left|\nabla V^{*}\right|_{1+\alpha} \leq B
$$

By (3.42) and (5.19)

$$
\left|\nabla \widetilde{V}_{1,1}\right|_{1+\alpha} \leq B \eta
$$

Estimates (5.26), (5.27) for $(n, i)=(1,1)$ with $B_{1}$ being replaced with $B$ follow from (3.34), (3.38)(3.41). Using (5.5), (5.26), (5.27) for $(n, i)=(1,1)$ with $B_{1}$ being replaced with $B$, as well as (3.20), (3.43), (5.9), (5.21), (5.35) and (5.36), we obtain

$$
\begin{aligned}
& \left|A_{1,1} \nabla \widetilde{V}_{1,1} \nabla q_{1}^{*}+A_{2,1} \nabla \widetilde{V}_{1,1}\left(\nabla V_{1,1}+\nabla V^{*}\right)-F_{1}\right|_{\alpha} \\
\leq & 8 \bar{s}^{2} B C^{*} \eta+16 \bar{s}^{2} B^{2} \eta+C^{*} \eta=8 \bar{s}^{2} B\left(2 B+C^{*}+\frac{C^{*}}{8 \bar{s}^{2}}\right) \eta \leq 4 \bar{s}^{2} B B_{1} \eta \leq \bar{s}^{2} B_{1}^{2} \eta .
\end{aligned}
$$

Thus,

$$
\left|A_{1,1} \nabla \widetilde{V}_{1,1} \nabla q_{1}^{*}+A_{2,1} \nabla \widetilde{V}_{1,1}\left(\nabla V_{1,1}+\nabla V^{*}\right)-F_{1}\right|_{\alpha} \leq \bar{s}^{2} B_{1}^{2} \eta
$$

By (5.21)

$$
C^{*}<\frac{B_{1}}{3}
$$

Next, consider coefficients in the left hand side of equation (5.33). Using (3.20) as well as (5.26) for $(n, i)=(1,1)$, we obtain $\left|A_{1,1} \nabla V_{1,1}\right|_{\alpha} \leq 8 \bar{s}^{2} B_{1}$. Hence, conditions (5.16) are satisfied with $P=8 \bar{s}^{2} B_{1}$. Hence, by (5.17) the solution of the Dirichlet boundary value problem (5.33), (5.34) can be estimated as

$$
\left|\widetilde{q}_{1,1}\right|_{2+\alpha} \leq \bar{s}^{2} K B_{1}^{2} \eta+K\left\|\widetilde{\psi}_{1}\right\|_{C^{2+\alpha}(\partial \Omega)}, K=K\left(\Omega, \bar{s}^{2} B_{1}\right)=\text { const. }>2 .
$$

Using (5.13), (5.20) and (5.38), we obtain from this inequality

$$
\left|\widetilde{q}_{1,1}\right|_{2+\alpha} \leq K B_{1}^{2}\left(\bar{s}^{2}+\frac{C^{*}}{B_{1}^{2}}\right) \eta \leq K B_{1}^{2}\left(\bar{s}^{2}+\frac{1}{72}\right) \eta \leq 2 \bar{s}^{2} K B_{1}^{2} \eta .
$$


Hence, applying (5.21), we obtain

$$
\left|\widetilde{q}_{1,1}\right|_{2+\alpha} \leq K B_{1}^{3} \eta
$$

Estimate (5.28) for $(n, i)=(1,1)$ follows from (5.39). Next, using (5.6), (5.23) and (5.39), we obtain (5.29) for $(n, i)=(1,1)$,

$$
\left|q_{1,1}\right|_{2+\alpha} \leq\left|\widetilde{q}_{1,1}\right|_{2+\alpha}+\left|q_{1}^{*}\right|_{2+\alpha} \leq K B_{1}^{3} \eta+C^{*} \leq 2 C^{*} .
$$

Since by (3.39) and (3.41) $V_{1,1} \in C^{2+\alpha}(\bar{\Omega})$, then (3.25) and (5.40) imply that $v_{1,1} \in C^{2+\alpha}(\bar{\Omega})$. Hence, by (3.26) $c_{1,1} \in C^{\alpha}(\bar{\Omega})$. This, (3.23) and (3.24) imply that $\bar{c}_{1,1} \in C^{\alpha}\left(\mathbb{R}^{3}\right)$. Hence, (5.24) is true for $(n, i)=(1,1)$.

Now we estimate the norm $\left|\widetilde{c}_{1,1}\right|_{\alpha}$. Subtracting (5.11) from (3.26) for $(n, i)=(1,1)$, we obtain

$$
\widetilde{c}_{1,1}=\Delta \widetilde{v}_{1,1}+s_{n}^{2} \nabla \widetilde{v}_{1,1}\left(\nabla v_{1,1}+\nabla v_{1}^{*}\right)-\bar{F}_{1} .
$$

Hence, using (5.12), (5.20), (5.38) and (5.41), we obtain

$$
\left|\widetilde{c}_{1,1}\right|_{\alpha} \leq\left|\nabla \widetilde{v}_{1,1}\right|_{1+\alpha}\left[1+\bar{s}^{2}\left(\left|\nabla v_{1,1}\right|_{\alpha}+\left|\nabla v_{1}^{*}\right|_{\alpha}\right)\right]+\frac{B_{1}}{3} \eta
$$

Subtracting (5.10) from (3.25), we obtain $\widetilde{v}_{1,1}=-h \widetilde{q}_{1,1}+\widetilde{V}_{1,1}$. Hence, it follows from (5.23), (5.27) at $(n, i)=(1,1)$ and from (5.39) that

$$
\left|\nabla \widetilde{v}_{1,1}\right|_{1+\alpha} \leq K B_{1}^{3} \eta^{2}+B_{1} \eta \leq 2 B_{1} \eta .
$$

By (5.5), (5.10), (5.21), (5.23) and (5.35) -(5.38)

$$
\left|\nabla v_{n}^{*}\right|_{1+\alpha} \leq C^{*} N \eta+B_{1} \leq 2 B_{1} .
$$

Hence, (5.43), (5.44) and (5.23) imply that

$$
\left|\nabla v_{1,1}\right|_{1+\alpha}=\left|\nabla \widetilde{v}_{1,1}+\nabla v_{1}^{*}\right|_{1+\alpha} \leq\left|\nabla \widetilde{v}_{1,1}\right|_{1+\alpha}+2 B_{1} \leq 2 B_{1} \eta+2 B_{1} \leq 3 B_{1} .
$$

Hence,

$$
1+\bar{s}^{2}\left(\left|\nabla v_{1,1}\right|_{\alpha}+\left|\nabla v_{1}^{*}\right|_{\alpha}\right) \leq 1+5 B_{1} \bar{s}^{2}<6 \bar{s}^{2} B_{1} .
$$

Hence, comparing (5.45) with (5.42) and (5.43), we obtain

$$
\left|\widetilde{c}_{1,1}\right|_{\alpha} \leq 12 \bar{s}^{2} B_{1}^{2} \eta+C^{*} \eta \leq B_{1}^{3} \eta .
$$

This establishes (5.30) for $(n, i)=(1,1)$. As it was proved above, (5.30) for $(n, i)=(1,1)$ implies (5.24) and (5.25) for $(n, i)=(1,1)$. In summary, we have established (5.24)-(5.30) for $(n, i)=(1,1)$.

Since we have established relations (5.24)-(5.30) for $(n, i)=(1,1)$, we can assume now that we have proved (5.24)-(5.30) for $\left(n^{\prime}, i^{\prime}\right) \in[0, n] \times[0, i-1]$, where $n \geq 1, i \geq 2$. We now want to prove (5.24)-(5.30) for $\left(n^{\prime}, i^{\prime}\right)=(n, i)$. The mathematical induction principle and formulas (3.29) and (3.30) imply that this would be sufficient for the proof of Theorem 5.1. 
Subtracting equation (5.8) from equation (3.28) and taking into account boundary conditions (see (5.7)), we obtain

$$
\begin{aligned}
& \Delta \widetilde{q}_{n, i}-A_{1, n}\left(h \sum_{j=0}^{n-1} \nabla q_{j}\right) \nabla \widetilde{q}_{n, i}+A_{1, n} \nabla V_{n, i} \cdot \nabla \widetilde{q}_{n, i} \\
&=\left(A_{1, n} \nabla q_{n}^{*}-A_{2, n} h \sum_{j=0}^{n-1}\left(\nabla q_{j}+\nabla q_{j}^{*}\right)+2 A_{2, n} \nabla V_{n, i}\right)\left(h \sum_{j=0}^{n-1} \nabla \widetilde{q}_{j}\right) \\
&+\left[2 A_{2, n} h \sum_{j=0}^{n-1} \nabla q_{j}^{*}-A_{1, n} \nabla q_{n}^{*}-A_{2, n}\left(\nabla V_{n, i}+\nabla V^{*}\right)\right] \nabla \widetilde{V}_{n, i}-F_{n}, \\
&\left.\widetilde{q}_{n, i}\right|_{\partial \Omega}=\widetilde{\psi}_{n}(x) .
\end{aligned}
$$

First, we estimate the difference of tails $\widetilde{V}_{n, i}$. Since relations (15.24)-(5.30) are valid for $\left(n^{\prime}, i^{\prime}\right) \in$ $[0, n] \times[0, i-1]$, then by Theorem 4.2

$$
\begin{aligned}
& \left|\nabla V_{n, i}\right|_{1+\alpha} \leq B_{1}, \\
& \left|\nabla \widetilde{V}_{n, i}\right|_{1+\alpha} \leq B\left|\widetilde{c}_{n, i-1}\right|_{\alpha} \leq B_{1} B_{1}^{3[i-1+(n-1) m]} \cdot \eta=B_{1}^{3[i-1+(n-1) m]+1} \cdot \eta . \\
& \left|\nabla \widetilde{V}_{n, i}\right|_{1+\alpha} \leq B\left|\widetilde{c}_{n, i-1}\right|_{\alpha} \leq B_{1} B_{1}^{3[i-1+(n-1) m]} \cdot \eta=B_{1}^{3[i-1+(n-1) m]+1} \cdot \eta .
\end{aligned}
$$

These estimates establish (5.26) and (5.27) for $\left(n^{\prime}, i^{\prime}\right)=(n, i)$.

We now estimate the right hand side of equation (5.47). First, using (3.20), (5.5), (5.35) and (5.48), we obtain

$$
\left|A_{1, n} \nabla q_{n}^{*}-A_{2, n} h \sum_{j=0}^{n-1}\left(\nabla q_{j}+\nabla q_{j}^{*}\right)+2 A_{2, n} \nabla V_{n, i}\right|_{\alpha} \leq 8 \bar{s}^{2}\left(C^{*}+3 C^{*} N h+2 B_{1}\right) \leq 8 \bar{s}^{2}\left(C^{*}+1+2 B_{1}\right) .
$$

This inequality and (5.38) lead to

$$
\left|A_{1, n} \nabla q_{n}^{*}-A_{2, n} h \sum_{j=0}^{n-1}\left(\nabla q_{j}+\nabla q_{j}^{*}\right)+2 A_{2, n} \nabla V_{n, i}\right|_{\alpha} \leq 24 \bar{s}^{2} B_{1}
$$

Estimates (5.28) hold for functions $\widetilde{q}_{j}=q_{j}-q_{j}^{*}, j \in[0, n-1]$. Hence, using (5.23), we obtain

$$
\left|h \sum_{j=0}^{n-1} \nabla \widetilde{q}_{j}\right|_{\alpha} \leq K B_{1}^{3 N m} N \eta^{2} \leq \eta
$$

Combining this with (5.50), we obtain the following estimate for the term in the second raw of (5.47)

$$
\left|A_{1, n} \nabla q_{n}^{*}-A_{2, n} h \sum_{j=0}^{n-1}\left(\nabla q_{j}+\nabla q_{j}^{*}\right)+2 A_{2, n} \nabla V_{n, i}\right|_{\alpha}\left|h \sum_{j=0}^{n-1} \nabla \widetilde{q}_{j}\right|_{\alpha} \leq 24 \bar{s}^{2} B_{1} \eta
$$


Next, using (3.20), (5.5), (5.21), (5.22), (5.35) and (5.48), we obtain

$$
\begin{aligned}
& \left|2 A_{2, n} h \sum_{j=0}^{n-1} \nabla q_{j}^{*}-A_{1, n} \nabla q_{n}^{*}-A_{2, n}\left(\nabla V_{n, i}+\nabla V^{*}\right)\right|_{\alpha} \\
\leq & 16 \bar{s}^{2} C^{*} N \eta+8 \bar{s}^{2} C^{*}+16 \bar{s}^{2} B_{1} \leq \bar{s}^{2}+3 \bar{s}^{2} B_{1}+16 \bar{s}^{2} B_{1} \leq 20 \bar{s}^{2} B_{1} .
\end{aligned}
$$

Hence, using (5.9), (5.38) and (5.49), we obtain

$$
\begin{aligned}
& \left.\left|2 A_{2, n} h \sum_{j=0}^{n-1} \nabla q_{j}^{*}-A_{1, n} \nabla q_{n}^{*}-A_{2, n}\left(\nabla V_{n, i}+\nabla V^{*}\right)\right|\right|_{\alpha}\left|\nabla \widetilde{V}_{n, i}\right|_{\alpha}+\left|F_{n}\right|_{\alpha} \\
\leq & 20 \bar{s}^{2} B_{1} B_{1}^{3[i-1+(n-1) m]+1} \cdot \eta+C^{*} \eta \leq 20 \bar{s}^{2} B_{1} B_{1}^{3[i-1+(n-1) m]+1} \eta+\frac{B_{1}}{3} \eta .
\end{aligned}
$$

Combining this with (5.51) and using (5.21), we obtain the following estimate for the right hand side (rhs) of (5.47)

$$
\begin{aligned}
|r h s|_{\alpha} & \leq 21 \bar{s}^{2} B_{1} B_{1}^{3[i-1+(n-1) m]+1} \cdot \eta+24 \bar{s}^{2} B_{1} \eta \leq 21 \bar{s}^{2} B_{1} B_{1}^{3[i-1+(n-1) m]+1}\left(1+\frac{24}{21 B_{1}}\right) \eta \\
& \leq 21 \bar{s}^{2} B_{1} B_{1}^{3[i-1+(n-1) m]+1}\left(1+\frac{1}{21}\right) \eta=22 \bar{s}^{2} B_{1} B_{1}^{3[i-1+(n-1) m]+1} \cdot \eta .
\end{aligned}
$$

Thus,

$$
|r h s|_{\alpha} \leq 22 \bar{s}^{2} B_{1} B_{1}^{3[i-1+(n-1) m]+1} \cdot \eta .
$$

We now estimate coefficients which are multiplied by $\nabla \widetilde{q}_{n, i}$ in the left hand side of (5.47). We use (3.20), (5.21), (5.22), (5.23) and (5.38). By the assumption of the mathematical induction method we have that inequalities (5.29) are valid for functions $q_{j}$ with $j \in[0, n-1]$. First,

$$
\left|A_{1, n}\left(h \sum_{j=0}^{n-1} \nabla q_{j}(x)\right)\right|_{\alpha} \leq 16 \bar{s}^{2} C^{*} N \eta \leq \frac{6 \bar{s}^{2}}{B_{1}^{3 N m}}<\frac{1}{10} .
$$

Next, using (3.20) and (5.48), we obtain

$$
\left|A_{1, n} \nabla V_{n, i}\right|_{1+\alpha} \leq 8 \bar{s}^{2} B_{1} .
$$

Hence, it follows from (5.53) and (5.54) that the Dirichlet boundary value problem (5.47) satisfies conditions (5.14)-(5.17) with $P=9 \bar{s}^{2} B_{1}, K=K\left(\Omega, \bar{s}^{2} B_{1}\right)>2$. Hence, using (5.13), (5.17), (5.20), (5.38) and (5.52), we obtain

$$
\left|\widetilde{q}_{n, i}\right|_{2+\alpha} \leq K\left[22 \bar{s}^{2} B_{1} B_{1}^{3[i-1+(n-1) m]+1}+C^{*}\right] \eta \leq K \cdot 23 \bar{s}^{2} B_{1} B_{1}^{3[i-1+(n-1) m]+1} \cdot \eta .
$$

Since by (5.21) $B_{1} \geq 24 \bar{s}^{2}$, then the last estimate leads to

$$
\left|\widetilde{q}_{n, i}\right|_{2+\alpha} \leq K B_{1}^{3[i+(n-1) m]} \cdot \eta
$$




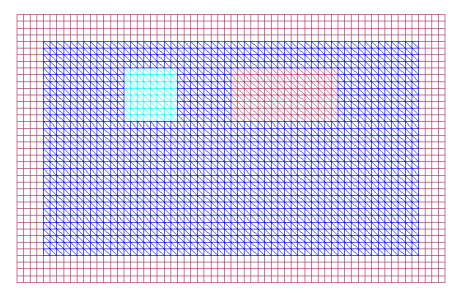

a) $G=G_{F E M} \cup G_{F D M}$

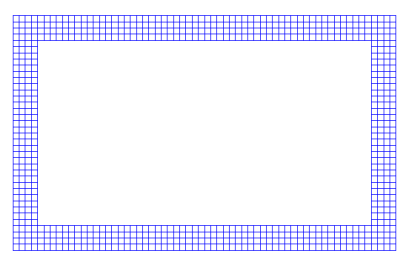

b) $G_{F D M}$

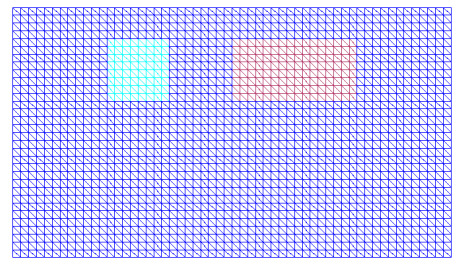

c) $G_{F E M}=\Omega$

FIG. 6.1. a) Geometry of the hybrid mesh. This is a combination of the quadrilateral mesh in the subdomain $G_{F D M} \mathrm{~b}$ ), where we apply FDM, and the finite element mesh in the inner domain $G_{F E M}=\Omega$ c), where we use FEM. The solution of the inverse problem is computed in $G_{F E M}=\Omega$.

which is (5.28). Next, we prove (5.29). We use (5.5), (5.6), (5.23) and (5.28),

$$
\left|q_{n, i}\right|_{2+\alpha} \leq\left|\widetilde{q}_{n, i}\right|_{2+\alpha}+\left|q_{n}^{*}\right|_{2+\alpha} \leq K B_{1}^{3[i+(n-1) m]} \cdot \eta+C^{*} \leq 2 C^{*} .
$$

Estimate now the norm $\left|\widetilde{c}_{n, i}\right|_{\alpha}$. We obtain similarly with (5.42)

$$
\left|\widetilde{c}_{n, i}\right|_{\alpha} \leq\left|\nabla \widetilde{v}_{n, i}\right|_{1+\alpha}\left[1+\bar{s}^{2}\left(\left|\nabla v_{n, i}\right|_{\alpha}+\left|\nabla v_{n}^{*}\right|_{\alpha}\right)\right]+\frac{B_{1}}{3} \eta
$$

We have

$$
\widetilde{v}_{n, i}(x)=-h \widetilde{q}_{n, i}(x)-h \sum_{j=0}^{n-1} \widetilde{q}_{j}(x)+\widetilde{V}_{n, i}(x), x \in \Omega .
$$

Hence, by (5.20), (5.21), (5.23), (5.27) and (5.28)

$$
\left|\nabla \widetilde{v}_{n, i}\right|_{1+\alpha} \leq K N B_{1}^{3[i+(n-1) m]} \eta^{2}+B_{1}^{3[i-1+(n-1) m]+1} \eta \leq \frac{25}{24} B_{1}^{3[i-1+(n-1) m]+1} \cdot \eta .
$$

Next, using (3.25) and (5.44), we obtain similarly with (5.45)

$$
1+\bar{s}^{2}\left(\left|\nabla v_{n, i}\right|_{\alpha}+\left|\nabla v_{n}^{*}\right|_{\alpha}\right) \leq 6 \bar{s}^{2} B_{1}
$$

Combining this with (5.55) and (5.56) and using (5.21), we obtain

$$
\begin{aligned}
\left|\widetilde{c}_{n, i}\right|_{\alpha} & \leq 7 \bar{s}^{2} B_{1} B_{1}^{3[i-1+(n-1) m]+1} \cdot \eta+\frac{B_{1}}{3} \eta \leq 8 \bar{s}^{2} B_{1} B_{1}^{3[i-1+(n-1) m]+1} \cdot \eta \\
& <B_{1}^{2} B_{1}^{3[i-1+(n-1) m]+1} \cdot \eta=B_{1}^{3[i+(n-1) m]} \cdot \eta .
\end{aligned}
$$

Thus, $\left|\widetilde{c}_{n, i}\right|_{\alpha} \leq B^{3[i+(n-1) m]} \eta$, which proves (5.30). Thus, relations (5.24) $-\left(\underline{5.30)}\right.$ are valid $\left(n^{\prime}, i^{\prime}\right)=$ $(n, i)$.

6. Numerical Studies. In this section we conduct some numerical experiments in both $2 \mathrm{~d}$ and $3 \mathrm{~d}$ cases. In the $2 \mathrm{~d}$ case we use specific ranges of parameters for a simplified mathematical model of imaging of antipersonnel land mines, see [28] and sections 6.8.2 and 6.8.3 of [6] for this model. In the $3 \mathrm{~d}$ case we model imaging of explosives hidden on belts worn by humans. We point 
out that in both cases our mathematical models are certainly simplified ones and further studies are necessary to see how they reflect the reality.

It is well known that there are always some discrepancies between the theories and numerical implementations of complicated numerical methods. We now list two discrepancies for our case. First, the above theory was developed for the case of the point source, because of a convenience of the analysis. In computations, however, we work with the case of an incident plane wave with the single direction of incidence. This is because it is better to operate with a plane wave computationally. Also, in the case when the point source is far from the domain of interest, it can be approximately treated as a plane wave. The above theory can be extended to the case of a plane wave after a purely technical additional effort. Second, to decrease the complexity of our computations, we replace (3.23) and (3.24) with the following simplified formula

$$
\bar{c}_{n, i}(x)=\left\{\begin{array}{c}
c_{n, i}(x) \text { if } c_{n, i}(x) \geq 1 \text { and } x \in \bar{\Omega} \\
1 \text { if either } c_{n, i}(x)<1 \text { or } x \notin \bar{\Omega} .
\end{array}\right.
$$

When reconstructing functions $c_{n, i}(x)$, we use a weak formulation of (3.26) via finite elements, see pages 184, 185 of [6] for this formulation. Comparison of Figures 3.12 and 3.13 of [6] (pages $182,183)$ shows that this formulation provides significantly more accurate results than the strong formulation (3.26).

We now describe our stopping criterion used in computations of sections 6.1 6.2. We stop computing functions $c_{n, i}$ on every pseudo-frequency interval $\left[s_{n}, s_{n-1}\right)$ when

$$
\text { either } \quad N_{n} \geq N_{n-1} \text { or } \quad N_{n} \leq \eta \text {, }
$$

where

$$
N_{n}=\frac{\left\|c_{n, i}-c_{n, i-1}\right\|_{L_{2}(\Omega)}}{\left\|c_{n, i}\right\|_{L_{2}(\Omega)}} .
$$

Here, $i$ is the number of iterations with respect to the tail on every pseudo-frequency interval $\left[s_{n}, s_{n-1}\right)$. Recall that we define by $m$ the number when iterations with respect to the tail are stopped.

To generate data for the CIP, we solve the forward problem for equation (3.2). Since it is impossible to numerically solve this problem in the entire space $\mathbb{R}^{n}(n=2,3)$, we solve it in a rectangle in 2-d and in a rectangular prism in 3-d, just as in [6]. We denote this each of these domains $G$. Thus, $G$ is our computational domain in which we compute the forward problem, and it replaces $\mathbb{R}^{n}(n=2,3)$. We impose the first order absorbing boundary condition [18] on one part of the boundary $\partial G$ and zero Neumann boundary condition on another part of $\partial G$. In all cases the domain of interest $\Omega \subset G, \partial \Omega \cap \partial G=\varnothing$, see for details below.

6.1. Our mathematical model of imaging of plastic antipersonnel land mines: 2d study. The first main simplification of our model is that we consider the $2 \mathrm{~d}$ case instead of $3 \mathrm{~d}$, although a $3 \mathrm{~d}$ numerical test is also presented below. Second, we ignore the air/ground interface, assuming that the governing PDE is valid on the entire $2 \mathrm{~d}$ plane. Results of studies of experimental data in [29, 30] as well as of section 6.9 of [6] indicate that the influence of the air/ground interface can be significantly decreased via a data pre-processing procedure.

Let the ground be

$$
\left\{\left(x_{1}, x_{2}\right): x_{2}<a=\text { const. }\right\} \subset \mathbb{R}^{2} .
$$


Consider a polarized electric field which is generated by a plane wave, initialized at the line $\left\{x_{2}=a^{0}>a, x_{1} \in \mathbb{R}\right\}$ at the moment of time $t=0$. The following hyperbolic equation can be derived from the Maxwell equations in the $2 \mathrm{~d}$ case

$$
c(x) u_{t t}=\Delta u,(x, t) \in \mathbb{R}^{2} \times(0, \infty) .
$$

where the function $u(x, t)$ is a component of the electric field and $c(x):=\varepsilon_{r}(x)$ is the spatially distributed dielectric constant. We assume that the function $c(x)$ satisfies conditions (3.1) in $2 \mathrm{~d}$. We model imaging of dielectric constants in plastic land mines. In doing so, we do not assume a knowledge of the background medium. So, images of land mines are constructed only on the basis of values of the dielectric constant $c(x)$ inside of them.

Let $\Omega$ be the domain of interest in the ground, where we search for land mines. We set

$$
\Omega=\{(x, y) \in(-0.35,0.35) \mathrm{m} \times(-0.05,0.35) \mathrm{m}\},
$$

where "m" stands for meter. Introducing dimensionless spatial variables $\left(x^{\prime}, y^{\prime}\right)=(x, y) /(0.1 \mathrm{~m})$ without changing notations for brevity, we obtain the dimensionless domain

$$
\Omega=(-3.5,3.5) \times(-0.5,3.5) .
$$

Hence, the ground is at $\left\{x_{2}=3.5\right\}$ and the depth of the domain of interest is 4 , which means 40 $\mathrm{cm}$ in real dimensions. Our backreflected signal is measured at the backscattering side,

$$
\text { the backscattering side is } \Gamma=\left\{\left(x_{1}, x_{2}\right): x_{1} \in(-0.35,0.35), x_{2}=3.5\right\} \text {. }
$$

It is well known that the maximal depth of an antipersonnel land mine does not exceed about 10 centimeters. Hence, we model these mines as two small rectangles with the $0.1 \mathrm{~m}$ and $0.2 \mathrm{~m}$ length of sides, and $0.1 \mathrm{~m}$ width of sides, respectively. Centers of those rectangles are located at $x_{2}=2.5$ which is of 10 depth $\mathrm{cm}$ in variables with dimensions, see Figure 6.1.

Tables of dielectric constants [38] show that in the dry sand the dielectric constant $\varepsilon_{r}=5$ and $\varepsilon_{r}=22$ in the trinitrotoluene (TNT). Hence, the mine/background contrast is $22 / 5 \approx 4$. Thus, we consider new parameters $\varepsilon_{r}^{\prime}, t^{\prime}$ without changing notations, $\varepsilon_{r}^{\prime}=\varepsilon_{r} / 5, t^{\prime}=t / \sqrt{5}$. Hence, we obtain the following relative values of the dielectric constant in our tests

$$
c(x)=\varepsilon_{r}(\text { dry sand })=1, c(x)=\varepsilon_{r}(\mathrm{TNT})=4 .
$$

6.2. Our mathematical model of imaging of explosives hidden in belts worn by humans: 3d study. In all places below where the $3 \mathrm{~d}$ case is discussed, we use the same notation for the vector $x=(x, y, z)$ and for its first coordinate. This does not lead to an ambiguity. In the $3 \mathrm{~d}$ case we model the body of a human as a rectangular prism of 2 meters tall, 0.6 meters wide and 0.16 meters "deep". The vertical coordinate is $y$ and $z$ is responsible for the depth. Hence, in this case computational domain $G$ is

$$
G=\{(x, y, z): x \in(-0.5,0.5), y \in(-1.08,1.08), z \in(-0.32,0.32)\} .
$$

We model the belt with explosives as the rectangular prism, which is a subdomain of the first one. Sizes of that "belt" are 0.3 meters in the vertical direction, 0.52 meters in horizontal direction and 0.08 meters of "depth". Hence, dividing by 1 meter, we obtain that these two prisms are respectively dimensionless domains $\Omega$ and $\Omega_{\text {belt }}$,

$$
\begin{aligned}
\Omega & =G_{F E M}=\{(x, y, z): x \in(-0.3,0.3), y \in(-1,1), z \in(-0.08,0.08)\}, \Omega \subset G \\
\Omega_{\text {belt }} & =\{(x, y, z): x \in(-0.26,0.26), y \in(-0.15,0.15), z \in(-0.04,0.04)\} \subset \Omega .
\end{aligned}
$$




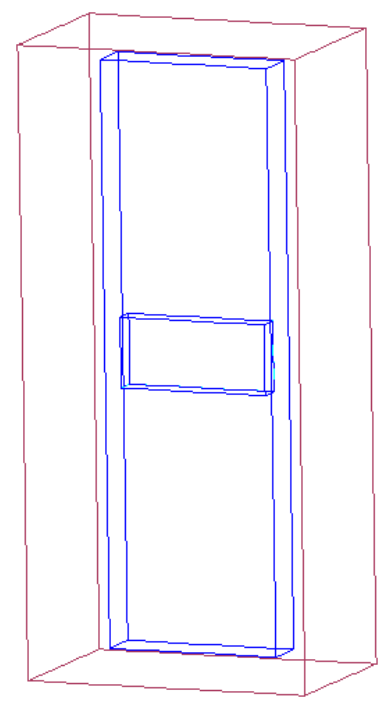

a)

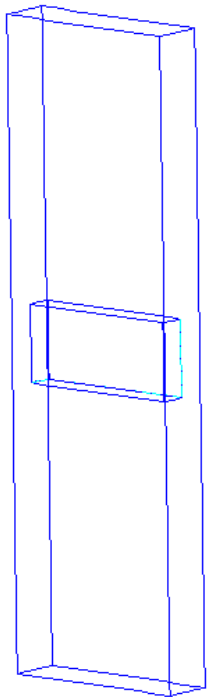

c)

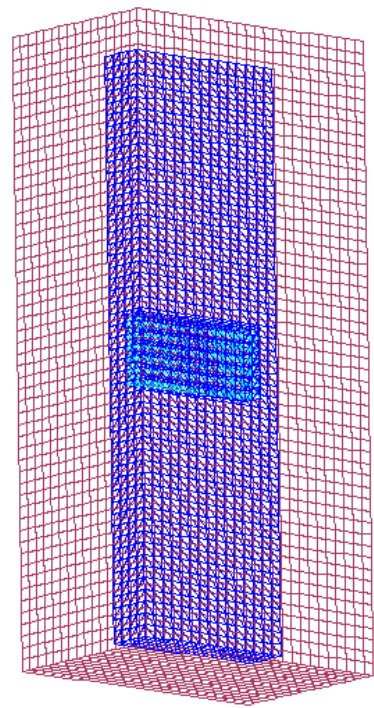

b)

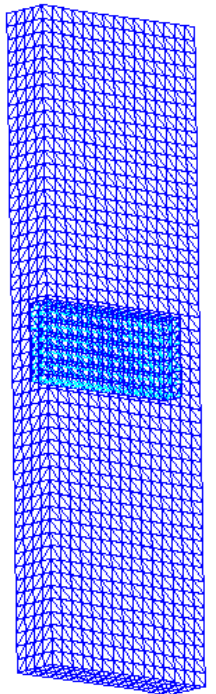

d)

Fig. 6.2. a) Hybrid FEM/FDM geometry $G$; b) Mesh outlined at the boundary in the Hybrid FEM/FDM geometry $G$; c) Inner FEM geometry $G_{F E M}=\Omega \subset G$; d) Mesh outlined at the boundary of the inner FEM geometry $G_{F E M}=\Omega$.

On Figure 6.2 the domain $G$ is the largest prism, $\Omega$ is the smaller prism and $\Omega_{\text {belt }}$ is the smallest prism. Our backscattering signal is measured at the front side $\Gamma$ of the prism $\Omega$. The incident plane wave propagates along the positive direction of the $z$-axis. Therefore, the front side of the prism 
$\Omega$ is the backscattering side. We define different boundaries of $G$ and $\Omega$ as

$$
\begin{aligned}
\text { Left side of } \Omega \text { is } \Gamma_{l} & =\{x=-0.3\} \cap \bar{\Omega}, \\
\text { Right side of } \Omega \text { is } \Gamma_{r} & =\{x=0.3\} \cap \bar{\Omega}, \\
\text { Back side of } \Omega \text { is } \Gamma_{b} & =\{z=0.08\} \cap \bar{\Omega}, \\
\text { Front (backscattering) side of } \Omega \text { is } \Gamma & =\{z=-0.08\} \cap \bar{\Omega}, \\
\text { Top side of } \Omega \text { is } \Gamma_{t} & =\{y=1\} \cap \bar{\Omega}, \\
\text { Bottom side of } \Omega \text { is } \Gamma_{b o t} & =\{y=-1\} \cap \bar{\Omega}, \\
\text { Front side of } G \text { is } \partial_{1} G & =\{z=-0.32\} \cap \bar{G}, \\
\text { Back side of } G \text { is } \partial_{2} G & =\{z=0.32\} \cap \bar{G}, \\
\partial_{3} G & =\partial G \backslash\left(\partial_{1} G \cup \partial_{2} G\right) .
\end{aligned}
$$

Therefore, we actually assume here that we measure the backreflected signal at the distance of $4 \mathrm{~cm}$ off the belt. Although this is unrealistic, we can justify this as follows. Suppose that we actually measure the backscattering signal on a plane $P=\{z=-Z, Z>0.08\}$. We can approximately assume that $w(x, y,-0.08, s)=w_{0}(x, y,-0.08, s)$ for $(x, y) \in \mathbb{R}^{2} \backslash \Gamma, s \in[\underline{s}, \bar{s}]$, see subsection 6.5. Recall that the function $\varphi(x, y, z, s)$ is the Laplace transform of the data $g(x, y, z, t)$ in (3.4) (subsection 3.2). Using the Green's function for the equation $\Delta w-s^{2} w=0$ in the half space $\{z<-0.08\}$, we can obtain an integral equation of the first kind with respect to the function $p(x, y, s):=w(x, y,-0.08, s)$. The right hand side of this equation will be the function $\varphi(x, y,-Z, s), s$ will be a parameter and integration will be carried out over the rectangle $\Gamma$. This is a convolution equation, which represents a linear ill-posed problem. Algorithms of solving convolution equations using the Tikhonov regularization are described in the book [39]. Thus, solution of this equation would provide us with an approximation of the function $w(x, y,-0.08, s)$ for $(x, y) \in \Gamma, s \in[\underline{s}, \bar{s}]$. On the other hand, the latter is the function which we consider as given data in our numerical experiments of subsection 6.9. Thus, assuming below that we have the data at $\Gamma$, we avoid the intermediate step of solving that integral equation.

6.3. Data simulation in 2 d. To simulate the data for our CIP in $2 d$, we solve the forward problem for equation (6.4) for the case of the incident plane wave propagating along the negative direction of the $x_{2}$ axis. This plane wave is initialized on the top boundary of the rectangle $G$ of Figure 6.1. We simulate the data for the inverse problem using the software package WavES [40]. To do that we solve the forward problem via the hybrid FEM/FDM method described in [5]. In this method the computational domain $G$ is split in two subdomains, $G=G_{F D M} \cup G_{F E M}$, where

$$
G=[-4,4] \times[-1,4], G_{F E M}:=\Omega=(-3.5,3.5) \times(-0.5,3.5), G_{F D M}=G \backslash G_{F E M},
$$

see Figure 6.1. Thus the subdomain $G_{F E M}:=\Omega$ is the same as in 6.5). We use structured mesh and FDM in $G_{F D M}$ and non-structured mesh and FEM in $G_{F E M}=\Omega$. The space mesh in $\Omega$ consists of triangles and it consists of squares in $G_{F D M}$, with the mesh size $\tilde{h}=0.125$ in the overlapping regions. At the top and bottom boundaries of $G$ we use first-order absorbing boundary conditions. These conditions are exact in our case since we initialize a plane wave in a normal direction to the top boundary of $G$. At the lateral boundaries, the zero Neumann boundary condition is used. Since the incident plane wave propagates downwards, then the zero Neumann boundary condition allows us to model an infinite space domain in the lateral direction. 


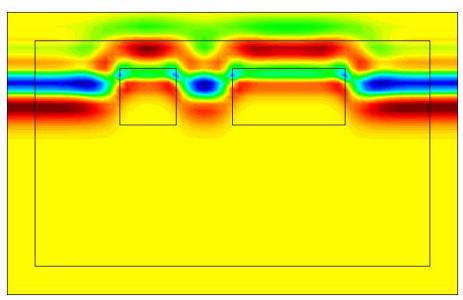

$\operatorname{step3}$

a) $\mathrm{t}=3.0$

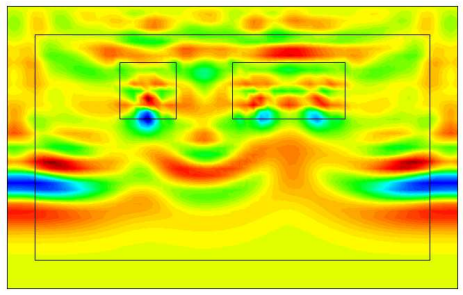

stop

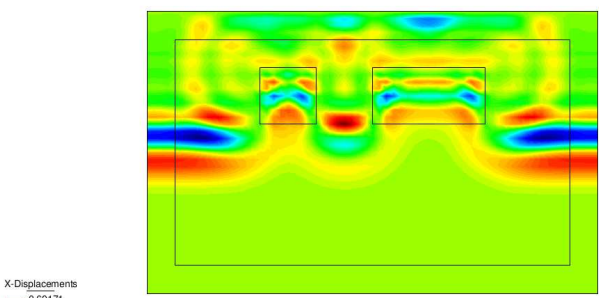

step 4

b) $\mathrm{t}=4.0$

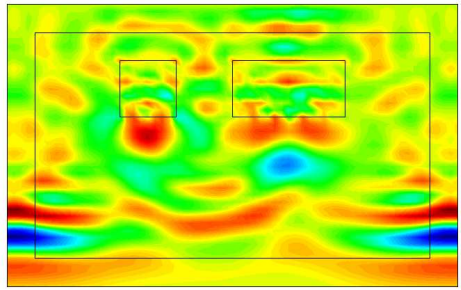

stepe

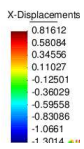

d) $\mathrm{t}=6.0$

FIG. 6.3. Isosurfaces of the computed solution $u(x, t)$ of the forward problem (6.21), 6.22) for the case of the mine-like targets of Figure 6.1 for different times. One can observe that values of $u(x, t)$ at the backscattering (top) side of the boundary are affected quite significantly by the presence of these targets. Also, values at the bottom side are significantly affected. However, since this side is located far away from the backscattering side, then the secondary reflected wave does not provide a significant impact on the backscattering side, see d). Values at lateral sides are almost the same as ones for the uniform background with $c(x) \equiv 1$. These observations are the basis for our decision about the change of boundary conditions, see (6.25).

Small square and small rectangle of Figure 6.1 are mine-like targets with $c(x)=4$ inside of them, see (6.7). Thus,

$$
c(x)=\left\{\begin{array}{c}
4 \text { in mine-like targets of Figure 6.1 } \\
1 \text { otherwise. }
\end{array}\right.
$$

When solving the inverse problem, we assume that the coefficient $c(x)$ is unknown in the rectangle $\Omega \subset G$ and has a known constant value $c(x)=1$ in $G \backslash \Omega$, see Figure 6.1 The boundary of the rectangle $G$ is $\partial G=\partial G_{1} \cup \partial G_{2} \cup \partial G_{3}$. Here, $\partial G_{1}$ and $\partial G_{2}$ are respectively top and bottom sides of the largest rectangle of Figure 6.1 and $\partial G_{3}$ is the union of left and right sides of this rectangle. Let $T$ be the final time for data generation, see the paragraph after (3.4) in subsection 3.1. We 
generate the data via solution of the following forward problem

$$
\begin{aligned}
c(x) u_{t t}-\Delta u & =0, \quad \text { in } G \times(0, T), \\
u(x, 0) & =0, u_{t}(x, 0)=0, \text { in } G, \\
\left.\partial_{n} u\right|_{\partial \Omega_{1}} & =f(t), \text { on } \partial G_{1} \times\left(0, t_{1}\right], \\
\left.\partial_{n} u\right|_{\partial \Omega_{1}} & =\partial_{t} u, \text { on } \partial G_{1} \times\left(t_{1}, T\right), \\
\left.\partial_{n} u\right|_{\partial G_{2}} & =\partial_{t} u, \text { on } \partial G_{2} \times(0, T), \\
\left.\partial_{n} u\right|_{\partial \Omega_{3}} & =0, \text { on } \partial G_{3} \times(0, T),
\end{aligned}
$$

The plane wave with the wave form $f(t)$ is initialized at the top boundary $\partial G_{1}$ of the computational domain $G$ during the time period $t \in(0,2 \pi / \omega]=\left(0, t_{1}\right]$, propagates downwards into $G$ and is absorbed at the bottom boundary $\partial G_{2}$ for all times $t \in(0, T)$. In addition, it is also absorbed at the top boundary $\partial G_{1}$ for times $t \in(2 \pi / \omega, T)$. Here

$$
f(t)=\left\{\begin{array}{c}
\frac{1}{10}(\sin (\omega t-\pi / 2)+1) \text { for } t \in\left(0, \frac{2 \pi}{\omega}\right] \\
0 \text { for } t \in\left(\frac{2 \pi}{\omega}, T\right)
\end{array}\right.
$$

We took $\omega=7$ and $T=6$ in (6.22) for $2 \mathrm{~d}$ tests. To update tails, we have solved on each iterative step the forward problem (6.21). Next, we have calculated the Laplace transform (3.5) to obtain the function $w_{n, i}(x, \bar{s})$, see (3.13) and (3.29).

The trace $g(x, t)$ of the solution $u(x, t)$ of the forward problem (6.21), 6.22) is recorded at the top boundary $\Gamma$ of the domain $\Omega$ where we solve the inverse problem, see (6.6). This trace generates the Dirichlet boundary data $\psi(x, s), x \in \Gamma$ in (3.16) (after the Laplace transform). Next, the coefficient $c(x)$ is "forgotten", and our goal is to reconstruct this coefficient for $x \in \Omega$ from the data $\psi(x, s)$.

6.4. Data simulation in 3d. In this case domains $G$ and $\Omega$ are those of (6.8) and (6.9) respectively. Since the human body consists mostly of water, and the dielectric constant of water is about 80 [38, we set in Test 5 below

$$
c(x)=\left\{\begin{array}{c}
80, x \in \Omega \backslash \Omega_{\text {belt }}, \\
1, x \in G \backslash \Omega, \\
3.2, x \in \Omega_{\text {belt }} .
\end{array}\right.
$$

Hence, 6.23) is a quite heterogeneous and, therefore, a very complicated case. Because of this, we start from a simpler problem in our Tests 3,4 via choosing

$$
c(x)=\left\{\begin{array}{c}
3.2, x \in \Omega_{\text {belt }}, \\
1, x \in G \backslash \Omega_{\text {belt }} .
\end{array}\right.
$$

As to the subdomain $\Omega_{\text {belt }} \subset \Omega$, we assume that it is filled with an improvised explosive device (IED). Analyzing dielectric constants of some materials which might form IEDs [16], we came to the conclusion that we can take $c(x)=3.2$ to model an IED. This value of the dielectric constant is close to RDX Hexahydro-1,3,5-trinitro-1,3,5-triazine [16. Given notations (6.9)-(6.19), (6.23), (6.24), we have simulated the data via solving the forward problem (6.21), (6.22). We have used the mesh step size $\widetilde{h}=0.04$ in $G$. 
In our $3 \mathrm{~d}$ tests we took $\omega=21$ and $T=1$ in (6.22). To generate backscattered data we solve the forward problem (6.21), (6.22) in time $t=[0,1]$ with the time step $\tau=0.001$ using the software package WavES [40. Figure 6.5 shows isosurfaces of the computed solution $u(x, t)$ of the forward problem (6.21), (6.22) for different times $t \in(0,1)$ for the case when the belt with explosives was as the one on Figure 6.2. The trace $g(x, t)$ of the solution $u(x, t)$ of the forward problem (6.21), (6.22) is recorded at the front boundary $\Gamma$ of the domain $\Omega$, which is the backscattering side of $\Omega$, see (6.14) for $\Gamma$. Again, this trace generates the Dirichlet boundary data $\psi(x, s), x \in \Gamma$ in (3.16) (after the Laplace transform). Next, the coefficient $c(x)$ is "forgotten", and our goal is to reconstruct this coefficient for $x \in \Omega$ from the data $\psi(x, s)$.

6.5. Boundary conditions on $\partial \Omega \backslash \Gamma$ and the choice of the $s$-interval in 2d. Although the above theory requires the knowledge of the function $u(x, t):=g(x, t)$ at the entire boundary $\partial \Omega$, the backscattering data are given only on the top part $\Gamma$ of the rectangle $\Omega$. To see how we can complement these data, we analyze the time dependent behavior of the function $u(x, t)$, which is calculated as the solution of the problem (6.21), 6.22). Figure 6.3 displays this function for different times $t \in(0,6)$ for all $x \in \Omega$ for the case of two mine-like targets of Figure 6.1 with $c(x)=4$ inside of them and $c(x)=1$ everywhere else, see (6.7). We see that values of $u(x, t)$ for $x \in G$ are substantially affected by the presence of these inclusions. On the other hand, values at lateral sides of the rectangle $\Omega$ are affected insignificantly. Values at the lower part of the boundary $\partial \Omega$ are also significantly affected by the presence of those targets. On the other hand, that lower part of $\partial \Omega$ is located rather far away from the top part of $\partial \Omega$. This means that waves reflected from the lower part reach the to part of $\partial \Omega$ at larger times $t>8$. On the other hand, the Laplace transform (3.5) actually discounts values of the function $u(x, t)$ for large $t$ : because of the rapid decay of the kernel $e^{-s t}$.

These observations provide a numerical justification for assigning the following boundary condition at $\partial \Omega$

$$
\left.w(x, s)\right|_{\partial \Omega}=\left\{\begin{array}{c}
w_{\text {calc }}(x, s), x \in \Gamma \\
w_{\text {unif }}(x, s), x \in \partial \Omega \backslash \Gamma
\end{array}\right.
$$

Here $w_{\text {calc }}(x, s)$ is the function $w(x, s)$ which is calculated as the Laplace transform (3.5) of the solution of the forward problem (6.21), (6.22). On the other hand $w_{\text {unif }}(x, s)$ is the the Laplace transform of the solution of this problem for the uniform medium with $c(x) \equiv 1$.

Consider now the function, $x \in \Omega$. Figure 6.4 displays graphs of the function $q(x, s)$ along the top boundary of the rectangle $\Omega$ for different values of the pseudo frequency $s$. One can observe that for $s=2,3,4,5,7$ each graph has dents. The locations of these dents exactly correspond to projections of two targets of Figure 6.1 on the top boundary of $\Omega$. Therefore, values of the function $q(x, s), x \in \Gamma$ carry an information about the horizontal coordinate of this inclusion. However, figuring out vertical coordinates of targets is a more difficult task. To do this, one needs to apply the above algorithm. We also observe that values of $|q(x, s)|$ for $s \geq 7$ are much lower than those for $s \leq 5$. Therefore, to solve the inverse problem in $2 \mathrm{~d}$, we have chosen the $s$-interval as

$$
s \in[2,3], h=0.05 .
$$

6.6. 3d case: boundary conditions on $\partial \Omega \backslash \Gamma$ and the choice of the $s$-interval. Just as in the $2 \mathrm{~d}$ case, we have chosen boundary conditions as in 6.25). To justify this, we present Figures 6.6.6.9. One can see from these figures that values of the function $w(x, s)$ at all parts of $\partial \Omega \backslash \Gamma$, except of the back side $\Gamma_{b}$ of the prism $\Omega$, are about the same as ones for the case $c(x) \equiv 1$, 
which is the value of this coefficient outside of our domain of interest $\Omega$. As to the surface $\Gamma_{b}$, it corresponds to the transmitted signal and values of $w(x, s)$ here are far from those of the uniform background outside of $\Omega$. However, just as in the $2 \mathrm{~d}$ case, the transmitted side $\Gamma_{b}$ is located far from the backscattering side $\Gamma$. Therefore, the Laplace transform (3.5) diminishes the influence of waves reflected from $\Gamma_{b}$, at least for large values of the parameter $s$, see Figures 6.6 6.7. Hence, the amplitude of reflected waves from this side is small when they reach $\Gamma$, compared with reflections at $\Gamma$ from the target $\Omega_{\text {belt }}$. This provides a numerical justification of (6.25) in the $3 \mathrm{~d}$ case. We call the resulting boundary function $\psi(x, s)$ "immersed boundary data", see Figure 6.11

6.7. Numerical tests for the $2 \mathbf{d}$ case. Let $u_{\text {calc }}(x, t), x \in \Gamma$ be the calculated solution of the forward problem (6.21), (6.22) at the backscattering side $\Gamma$ of the boundary $\partial \Omega$. We have introduced a random noise in the function $u_{\text {calc }}(x, t), x \in \Gamma$ as

$$
u_{\sigma}\left(x^{(i)}, t^{(j)}\right)=u_{\text {calc }}\left(x^{(i)}, t^{(j)}\right)\left[1+\alpha_{j} \sigma\left(u_{\max }-u_{\min }\right)\right],
$$

where $\left(x^{(i)}, t^{(j)}\right) \in \Gamma \times(0, T)$ are mesh points, $u_{\max }$ and $u_{\min }$ are maximal and minimal values of $u_{\text {calc }}(x, t)$ for $x \in \Gamma$, numbers $\alpha_{j} \in(-1,1)$ are randomly distributed and $\sigma=0.05$. Thus, the noise level was 5\%. In both Test 1 and Test 2 the correct coefficient $c(x)$ is the same as in (6.20).

Test 1. In this test the initial guess for the tail function $V_{1,1}$ the function was computed via (3.13) for the case of the homogeneous domain $G$ with $c(x) \equiv 1$. Next, the algorithm of subsection 3.4 was applied to reconstruct the true function $c(x)$ in (6.20). The computed image is presented on Figure 6.12 a). We observe that both the location and the contrast of both mine-like targets are reconstructed accurately. The number of inner iterations with respect to tails was $m=5$. The stopping criterion (6.2)-(6.3) was achieved at $c_{3,5}(x)$, i.e. we have stopped at $n=3$. By (6.26), (3.18) and (3.19) this corresponds to $s \in[2.85,2.90]$. The reconstructed dielectric constant in this test is $c_{3,5}(x)=4.07$ inside of both imaged mine-like targets and $c_{3,5}(x)=1$ at all other points of $\Omega$. To see what happens in an ideal case when the exact tail function $V^{*}(x)$ is known, we refer to Figure 6.12 $\mathrm{b}$ ), which corresponds to the function $c_{1,1}(x)$. Figure 6.12-b) confirms that the reconstruction is perfect in this case.

Test 2. In this test we choose the initial guess for the tail function $V_{1,1}$ as an initial guess for the tail function $V_{1,1}$ we take the function computed via (3.41) and use the algorithm of subsection 3.4 to reconstruct the dielectric constant of Figure $6.13 \mathrm{ra}$ ). In this test the reconstructed dielectric constant is $c_{8,6}(x)=4.27$ inside mine-like targets and $c_{8,6}(x)=1$ at all other points of $\Omega$. This reconstruction was obtained on the pseudo-frequency interval $s \in[2.6,2.65]$ and after 6 iterations with respect to the tail function. In other words, we took the number of inner iterations with respect to tails $m=6$ and the stopping criterion (6.2)-(6.3) was achieved at $n=8$.

Thus, in both Tests 1,2 reconstructions were accurate ones.

6.8. Numerical test for the $\mathbf{3 d}$ case. In Test 3 and Test 4 we present results for the case (6.24), and in Test 5 - for the case (6.23). The same random noise of $5 \%$ was introduced as the one in (6.27).

Test 3. In this test we took the first guess for the tail function $V_{1,1}(x)$ the same as the one for the uniform background when $c(x) \equiv 1$ for $x \in G$. Using Figures 6.6, 6.7 and analyzing the backscattered data $\psi(x, s)$ for $x \in \Gamma$ we have decided to choose the interval of pseudo frequencies as

$$
s \in[4,11], h=1 .
$$


Then we have used the algorithm of subsection 3.4 to reconstruct the dielectric constant in the belt of Figure 6.2 c). Figure 6.14 a) presents reconstruction of the dielectric constant $c(x)$ for the unrealistic case when we know the exact tail function. In this case we observe that the reconstruction is perfect.

Figure 6.14-b) shows the maximal values of the reconstructed function $c(x)$ when the initial tail $V_{1,1}(x)$ was computed from the homogeneous domain with $c(x) \equiv 1$ for $x \in G$. We observe that the location and the contrast of the explosive-like target are reconstructed accurately. The reconstructed dielectric constant in this test is $c_{3,2}(x)=3.54$ inside the "belt", and $c(x)=1$ at all other points of $\Omega$. We took the number of inner iterations with respect to tails $m=2$, and the stopping criterion (6.2)-(6.3) was achieved at $n=3$, which corresponds to $s \in[8,9]=\left[s_{4}, s_{3}\right]$ in (3.18), (3.19). We conclude that this reconstruction is accurate.

Test 4. In this test we took the tail $V_{1,1}(x)$ the same as in our above theory, see (3.39), (3.40), (3.41). Analyzing results of Test 3 we have also decided to refine the pseudo-frequency interval in this test. Indeed, we got our final image of Test 3 for $s \in[8,9]$. Hence, we decided to take the interval of pseudo frequencies

$$
s \in[8,8.85], h=0.05 .
$$

Next, we have used the algorithm of subsection 3.4. We took $m=2$. The stopping criterion (6.2)-(6.3) was achieved at $n=3$, which corresponds to $s \in[8.7,8.75]=\left[s_{4}, s_{3}\right]$ in (3.18), (3.19). The reconstructed function $c_{3,2}(x)$ is depicted on Figure 6.15-a). In this test the reconstructed dielectric constant is $c_{3,2}(x)=3.54$ inside the belt and $c_{3,2}(x)=1$ at all other points of $\Omega$. Thus, the reconstruction was again a quite accurate one.

Test 5. This is the most challenging test, because the medium is a quite heterogeneous one: there are substantial contrasts between three values of the target function $c(x)$. Indeed, we have used the case (this test we took the first guess for the tail $V_{1,1}(x)$ as in our above theory see (3.39), ( (3.41), i.e. the same as the one in Test 4. Used results of Test 4 we took now the interval of pseudo-frequencies

$$
s \in[8.0,8.8], h=0.05 .
$$

Next, we have used the algorithm of subsection 3.4. However, since we know that the dielectric constant of the human body is large, $c=80$, then we have truncated to 1 those values of computed functions $c_{n, i}(x)$, which exceeded 10. In other words (6.1), was replaced with

$$
\bar{c}_{n, i}(x)=\left\{\begin{array}{c}
c_{n, i}(x) \text { if } c_{n, i}(x) \in[1,10] \text { and } x \in \bar{\Omega} \\
1 \text { if either } c_{n, i}(x)<1, \text { or } c_{n, i}(x)>10, \text { or } x \notin \bar{\Omega} .
\end{array}\right.
$$

We took $m=3$ and the stopping criterion (6.2)- (6.3) was achieved at $n=2$. The latter corresponds to $s \in[8.70,8.75]=\left[s_{3}, s_{2}\right]$ in (3.18), (3.19). The reconstructed function $c_{2,3}(x)$ is depicted on Figure 6.15-b). The reconstructed dielectric constant is $c=3.09$ inside the belt, and $c=1$ at all other points of $\Omega$. Therefore, the reconstruction is again a quite accurate one even in this most difficult case.

7. Summary. We have presented a new approximate mathematical model. This model amounts to the truncation of the asymptotic series with respect to $1 / \bar{s}$, where $\bar{s}>>1$ is the upper limit of the positive parameter of the Laplace transform of the solution of the Cauchy problem (3.2), (3.3). However, this truncation is done only on the first iteration of our method to ensure 
estimate (5.36) for the accuracy of the first tail function $\left|\nabla \widetilde{V}_{1,1}\right|_{1+\alpha}$. No other "special" simplifying assumptions are made. On the basis of this new model, we have developed a new convergence analysis, which is more realistic than the one of our first publications [7, 8] about this topic. This time we estimate tail functions. Tails were not estimated in our previous publications, and this is a significantly new element of the convergence analysis here.

We have modified our approximately globally convergent algorithm for the case of backscattering data. To do so, we have used a computational observation that one can replace the unknown Dirichlet boundary condition on the non-backscattering part of the boundary with the data obtained for the case of the uniform background, which is assumed to be known outside of the domain of interest (but not inside of it), see (6.25). Therefore, our previously developed technique for the case when the Dirichlet data are given at the entire boundary, works. Our numerical tests confirm this.

Our numerical tests 1-4 demonstrate that the case when the first tail is taken the same as the one for the uniform medium with $c(x) \equiv 1$ provides almost the same results as ones for the new tail function. Numerical studies demonstrate the accuracy of our technique. It is worthy to note that we have obtained an accurate image even in the most difficult case of Test 5 when the medium was quite a heterogeneous one, see 6.23).

We believe that results of Tests 1-5 combined with results for blind experimental data of [29, 30] and section 6.9 of [6] confirm the validity of our approximate mathematical model, as indicated in Steps 4-6 of section 2 .

\section{Acknowledgments}

This research was supported by US Army Research Laboratory and US Army Research Office grant W911NF-11-1-0399, the Swedish Research Council, the Swedish Foundation for Strategic Research (SSF) in Gothenburg Mathematical Modelling Centre (GMMC) and by the Swedish Institute, Visby Program.

\section{REFERENCES}

[1]

[2] N.V. Alexeenko, V.A. Burov and O.D. Rumyantseva, Solution of a three-dimensional acoustical inverse scattering problem: II. Modified Novikov algorithm, Acoustical Physics, 54, 407-419, 2008.

[3] M. Asadzadeh and L. Beilina, A posteriori error analysis in a globally convergent numerical method for a hyperbolic coefficient inverse problem, Inverse Problems, 26, 115007, 2010.

[4] A.B. Bakushinskii and M.Yu. Kokurin, Iterative Methods for Approximate Solution of Inverse Problems, Springer, New York, 2004.

[5] L. Beilina, K. Samuelsson and K. Åhlander, Efficiency of a hybrid method for the wave equation. In International Conference on Finite Element Methods, Gakuto International Series Mathematical Sciences and Applications, Gakkotosho CO., LTD, 2001.

[6] L. Beilina and M.V. Klibanov, Approximate Global Convergence and Adaptivity for Coefficient Inverse Problems, Springer, New York, 2012.

[7] L. Beilina and M.V. Klibanov, A globally convergent numerical method for a coefficient inverse problem, SIAM J. Sci. Comp., 31, 478-509, 2008.

[8] L. Beilina and M.V. Klibanov, Synthesis of global convergence and adaptivity for a hyperbolic coefficient inverse problem in 3D, J. Inverse and Ill-posed Problems, 18, 85-132, 2010.

[9] L. Beilina and M.V. Klibanov, A posteriori error estimates for the adaptivity technique for the Tikhonov functional and global convergence for a coefficient inverse problem, Inverse Problems, 26, 045012, 2010.

[10] L. Beilina and M.V.Klibanov, Reconstruction of dielectrics from experimental data via a hybrid globally convergent/adaptive inverse algorithm, Inverse Problems, 26, 125009, 2010.

[11] L. Beilina, M.V. Klibanov and M.Yu Kokurin, Adaptivity with relaxation for ill-posed problems and global convergence for a coefficient inverse problem, Journal of Mathematical Sciences, 167, 279-325, 2010. 
[12] L. Beilina, Energy estimates and numerical verification of the stabilized domain decomposition finite element/finite difference approach for the Maxwell's system in time domain, Central European Journal of Mathematics, accepted for publication; preprint is available online at http://publications.lib.chalmers.se/publication/142368

[13] A.L. Bukhgeim and M.V. Klibanov, Uniqueness in the large of a class of multidimensional inverse problems, Soviet Math. Doklady, 17, 244-247, 1981.

[14] A.L. Bukhgeim, Carleman estimates for Volterra operators and uniqueness of inverse problems, in Non-Classical Problems of Mathematical Physics, pages 54-64, published by Computing Center of the Siberian Branch of USSR Academy of Science, Novosibirsk, 1981 (in Russian).

[15] A.L. Bukhgeim, Introduction in the Theory of Inverse Problems, VSP, Utrecht, The Netherlands, 2000.

[16] D. J. Daniels, UWB radar for the detection of buried ordnance, NATO/OTAN, unclassified/unlimited, can be found online at ftp.rta.nato.int/public/PubFullText/RTO/... \$MP-SET-120-KN.doc

[17] H.W. Engl, M. Hanke and A. Neubauer, Regularization of Inverse Problems, Kluwer Academic Publishers, Boston, 2000.

[18] B. Engquist and A. Majda, Absorbing boundary conditions for the numerical simulation of waves Math Comp. 31, 629-651, 1977.

[19] S.I. Kabanikhin, A.D. Satybaev and M.A. Shishlenin, Direct Methods for Solving Multidimensional Inverse Hyperbolic Problems, VSP, Utrecht, The Netherlands, 2004.

[20] S.I. Kabanikhin and M.A. Shishlenin, Numerical algorithm for two-dimensional inverse acoustic problem based on Gel'fand-Levitan-Krein equation, J. Inverse and Ill-Posed Problems, 18, 979-995, 2011.

[21] M. V. Klibanov, Uniqueness of solutions in the 'large' of some multidimensional inverse problems, in Non-Classical Problems of Mathematical Physics, pages 101-114, 1981, published by Computing Center of the Siberian Branch of the USSR Academy of Science, Novosibirsk (in Russian).

[22] M. V. Klibanov, Inverse problems in the 'large' and Carleman bounds, Differential Equations, 20, 755-760, 1984.

[23] M. V. Klibanov, Inverse problems and Carleman estimates, Inverse Problems, 8, 575-596, 1992.

[24] M. V. Klibanov and A. Timonov, Carleman Estimates for Coefficient Inverse Problems and Numerical Applications, VSP, Utrecht, 2004.

[25] M. V. Klibanov, M. A. Fiddy, L. Beilina, N. Pantong and J. Schenk, Picosecond scale experimental verification of a globally convergent numerical method for a coefficient inverse problem, Inverse Problems, 26, 045003, 2010.

[26] M.V. Klibanov, A.B. Bakushinskii and L. Beilina, Why a minimizer of the Tikhonov functional is closer to the exact solution than the first guess, J. Inverse and Ill-Posed Problems, 19, 83-105, 2011.

[27] M.V. Klibanov, J. Su, N. Pantong, H. Shan and H. Liu, A globally convergent numerical method for an inverse elliptic problem of optical tomography, Applicable Analysis, 89, 861-891, 2010.

[28] A. V. Kuzhuget, L. Beilina and M. V. Klibanov, Approximate global convergence and quasi-reversibility for a coefficient inverse problem with backscattered data, Journal of Mathematical Sciences, 181, 19-49, 2012.

[29] A.V. Kuzhuget, L. Beilina, M.V. Klibanov, A. Sullivan, L. Nguyen and M.A. Fiddy, Blind experimental data collected in the field and an approximately globally convergent inverse algorithm, Inverse Problems, 28, 095007, 2012.

[30] A.V. Kuzhuget, L. Beilina, M.V. Klibanov, A. Sullivan, L. Nguyen and M.A. Fiddy, Quantitative image recovery from measured blind backscattered data using a globally convergent inverse method, IEEE Transactions of Geoscience and Remote Sensing, accepted for publication, to be published in 2012.

[31] O. A. Ladyzhenskaya and N. N. Uralceva, Linear and Quasilinear Elliptic Equations, Academic Press, New York, 1969.

[32] R.G. Novikov, The $\partial$-bar approach to approximate inverse scattering at fixed energy in three dimensions, Int. Math. Res. Papers, 6, 287-349, 2005.

[33] R.G. Novikov and M. Santacesaria, Monochromatic reconstruction algorithms for two-dimensional multi-channel inverse problems, International Mathematics Research Notices, to appear.

[34] V.G. Romanov, Inverse Problems of Mathematical Physics, VNU, Utrecht, The Netherlands, 1986.

[35] V.G. Romanov, Investigation Methods for Inverse Problems, VSP, Utrecht, The Netherlands, 2002.

[36] Y. Shestopalov and Y. Smirnov, Determination of permittivity of an inhomogeneous dielectric body in a waveguide, Inverse Problems, 27, 095010, 2011.

[37] J. Su, M. V. Klibanov, Y. Liu, Z. Lin, N. Pantong and H. Liu, Optical imaging of phantoms from real data by an approximately globally convergent inverse algorithm, Arxiv 1208.5175v1 [math-ph], 25 Aug. 2012.

[38] Tables of dielectric constants at http://www.asiinstr.com/technical/Dielectric/020Constants.htm.

[39] A.N. Tikhonov, A.V. Goncharsky, V.V. Stepanov and A.G. Yagola, Numerical Methods for the Solution of Ill-Posed Problems, London: Kluwer, London, 1995. 
[40] WavES, the software package, http://www.waves24.com 


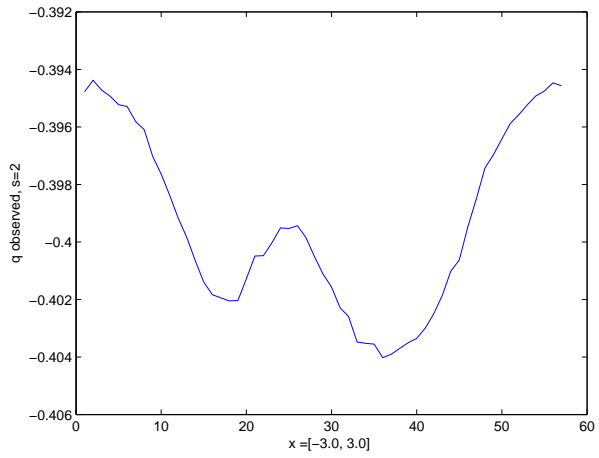

a) $s=2$

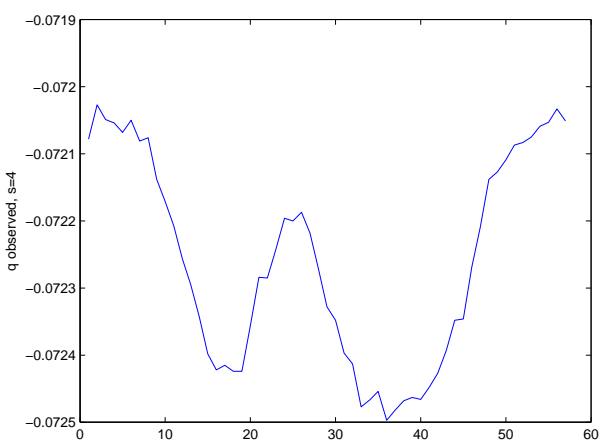

c) $s=4$

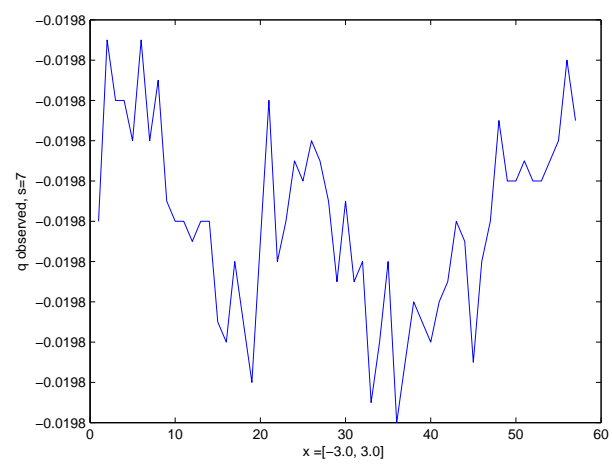

a) $s=7$

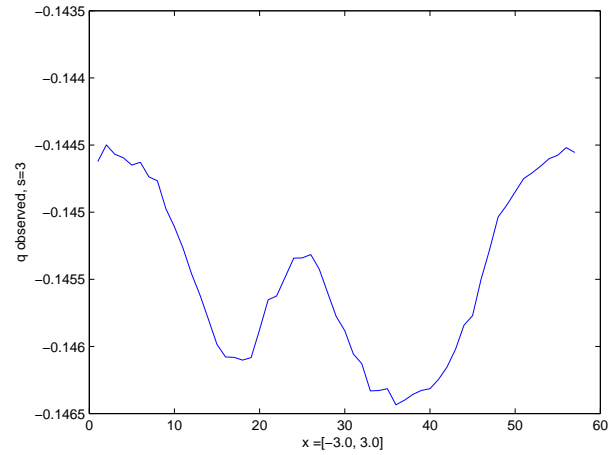

b) $s=3$

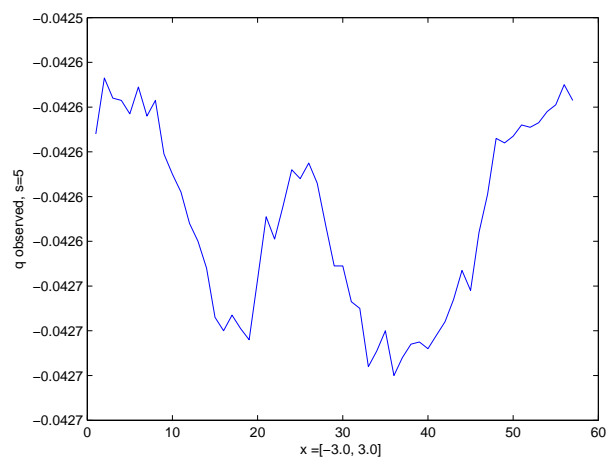

d) $s=5$

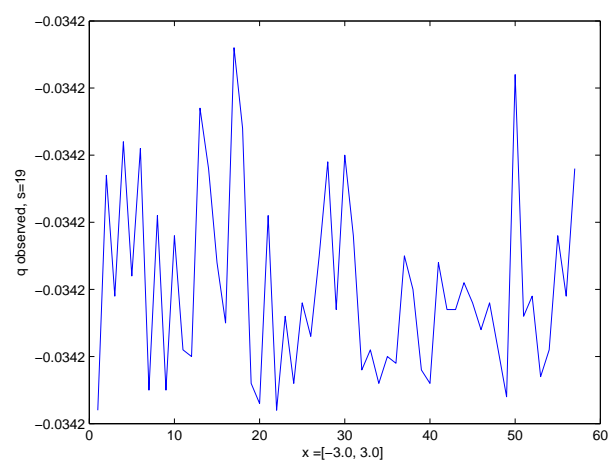

b) $s=19$

FIG. 6.4. Backscattered data at the top boundary $\Gamma$ of the function $q(x, s)$ at different values pseudo-frequencies $s$. 


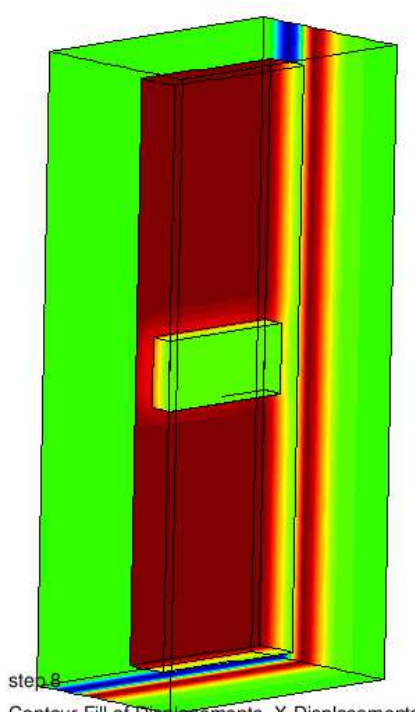

a) $t=0.4$

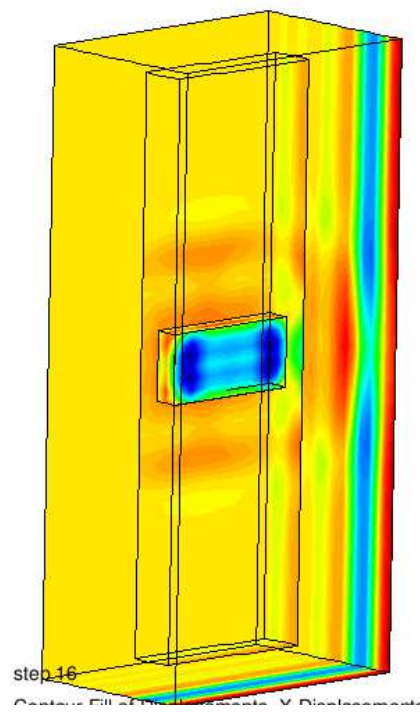

a) $t=0.8$

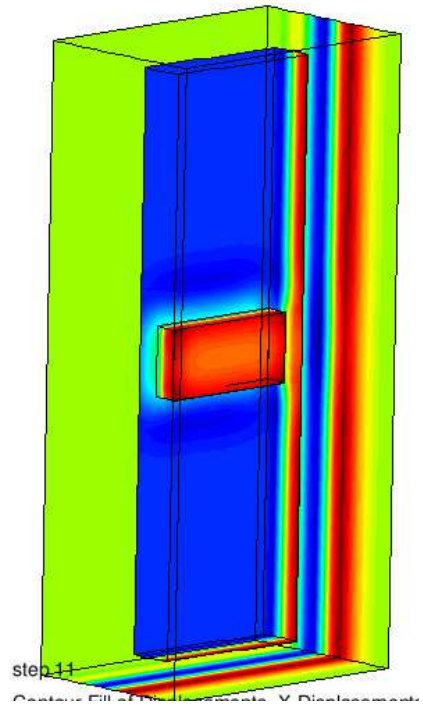

b) $t=0.55$

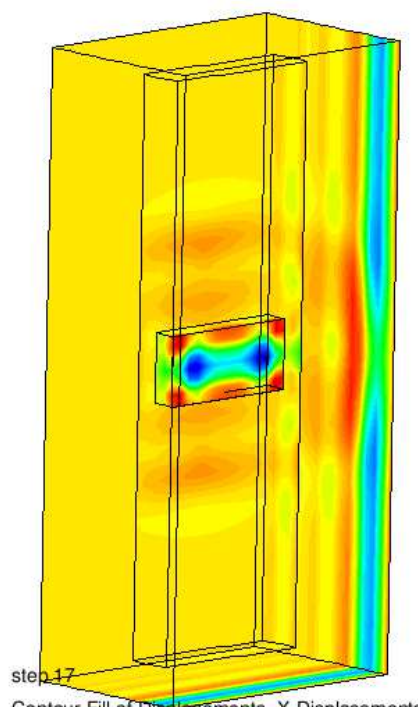

b) $t=0.85$

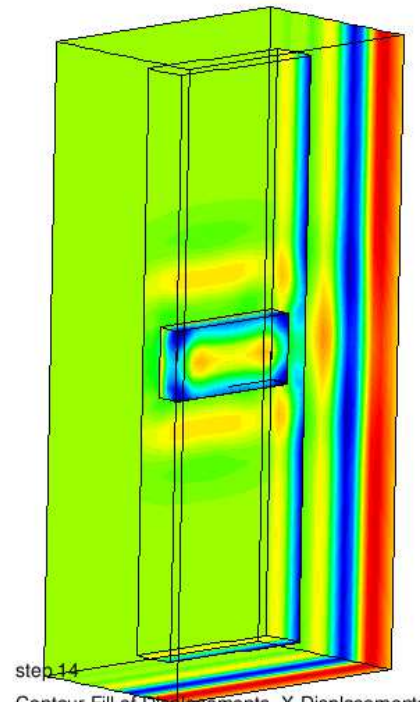

c) $t=0.7$

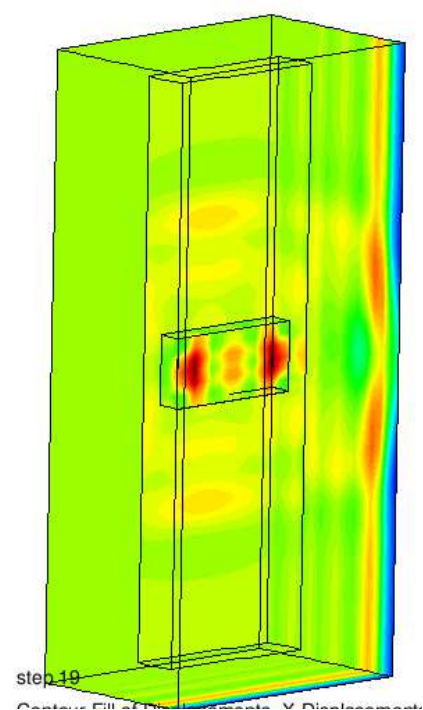

c) $t=0.95$

FIG. 6.5. Isosurfaces of the computed solution $u(x, t)$ of the forward problem (6.21), 6.22) at different times $t$ with the plane wave initialized at the front boundary of $G$ on the mesh with the mesh size $h=0.04$. Test was performed in time $t=[0,1]$ with time step $\tau=0.001$. 


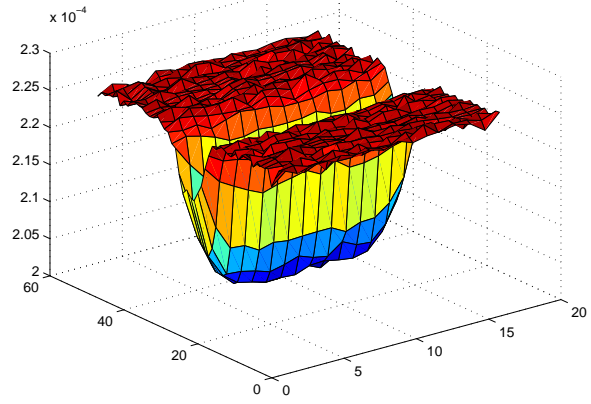

a) $s=20$

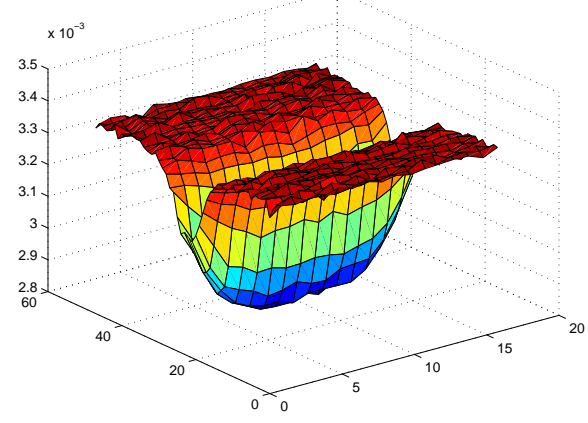

c) $s=10$

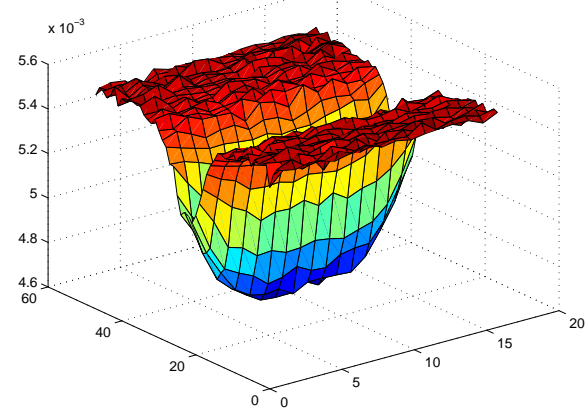

a) $s=8$

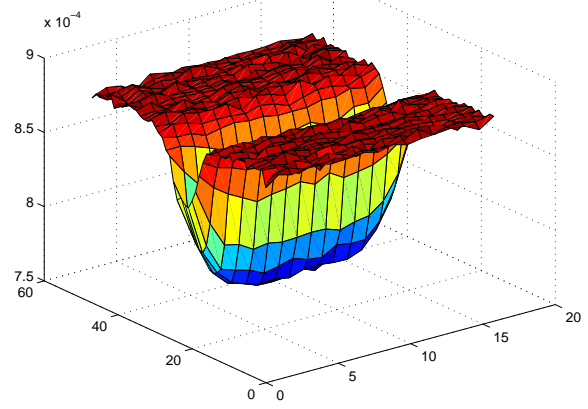

b) $s=15$

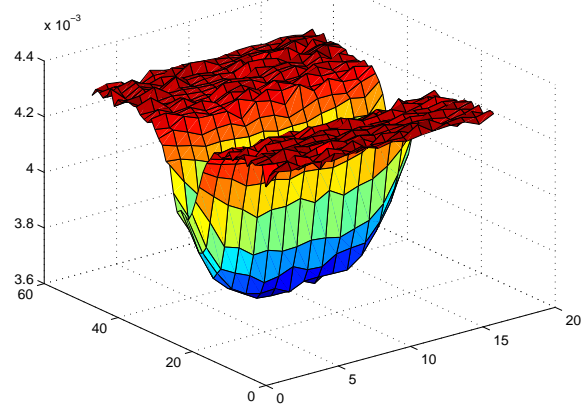

d) $s=9$

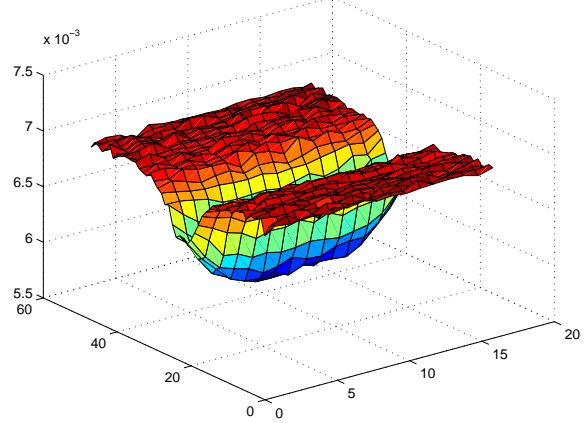

b) $s=7$

FIG. 6.6. Backscattered data $\psi(x, s), x \in \Gamma$ at different pseudo-frequencies $s \in[7 ; 20]$. 


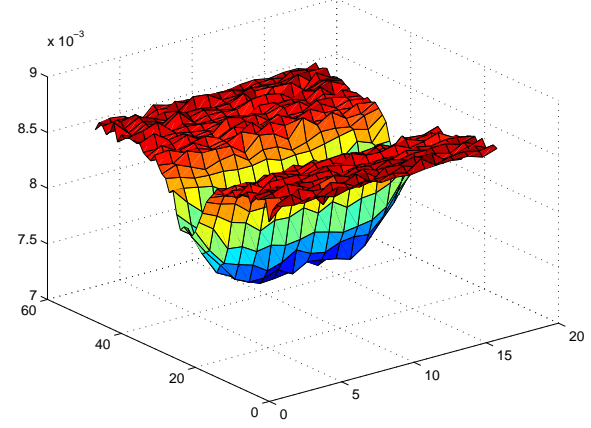

a) $s=6$

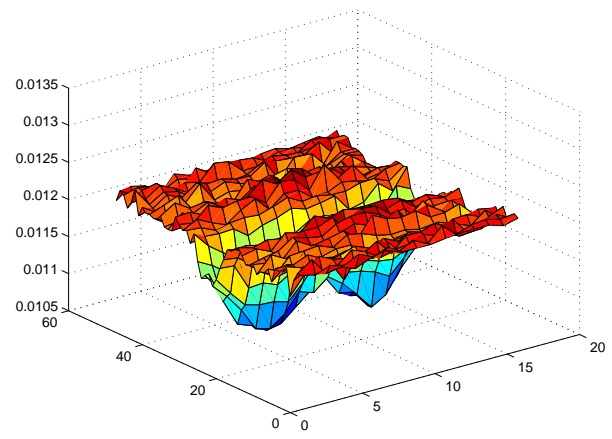

c) $s=4$

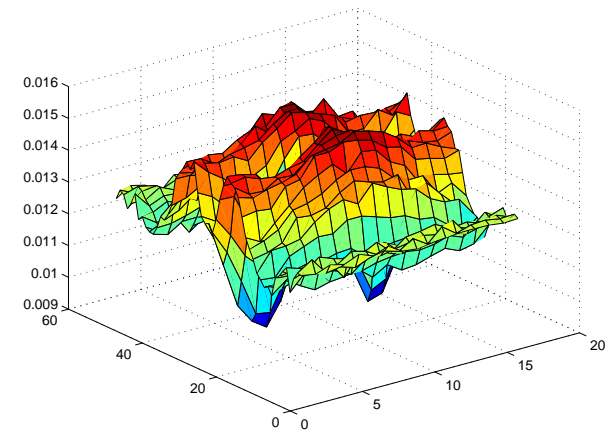

a) $s=2$

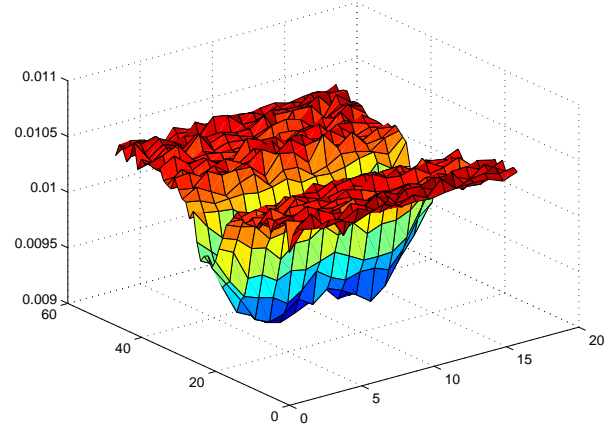

b) $s=5$

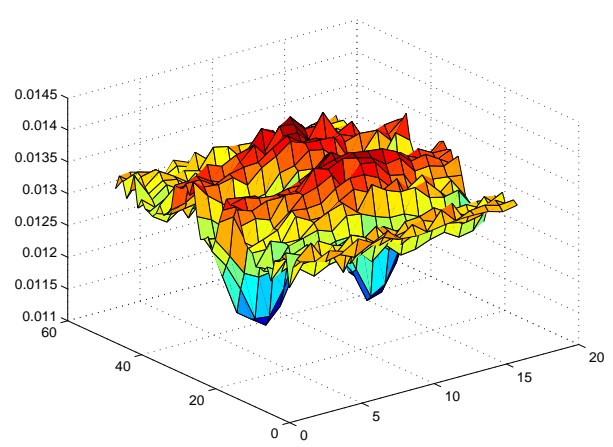

d) $s=3$

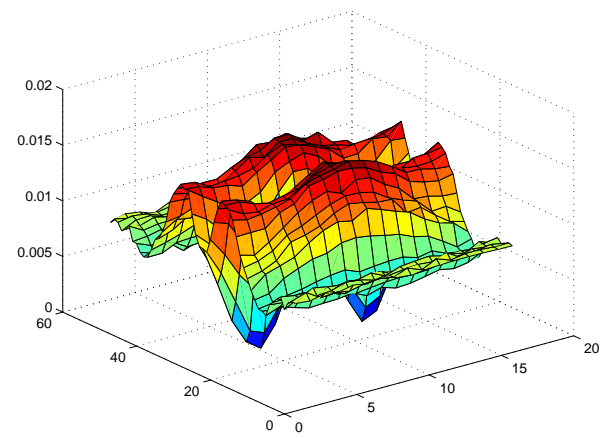

b) $s=1$

Fig. 6.7. Backscattered data $\psi(x, s), x \in \Gamma$ at different pseudo-frequencies $s \in[1,6]$. One can see from Figures 6.6 and 6.7 that one should take $s \geq 4$ in the reconstruction algorithm in $3 \mathrm{~d}$ tests. 


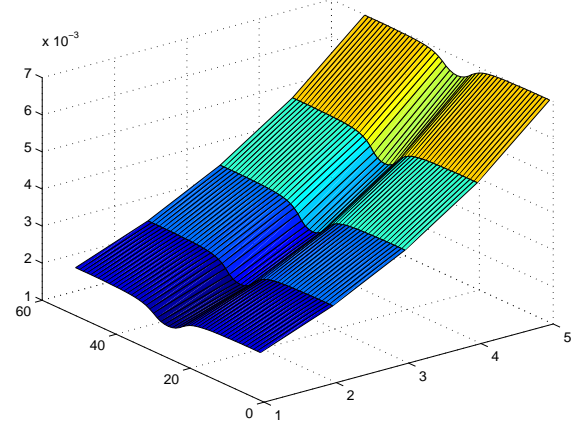

a)

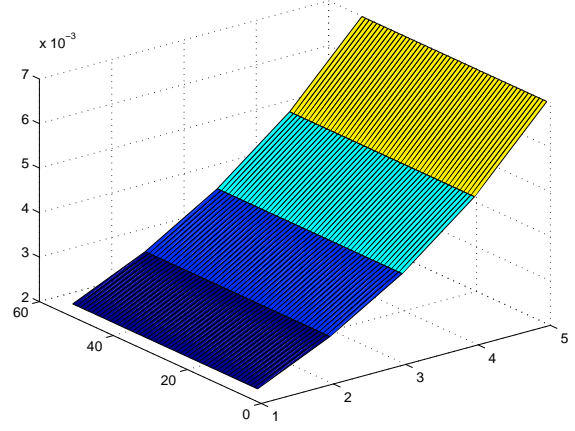

b)

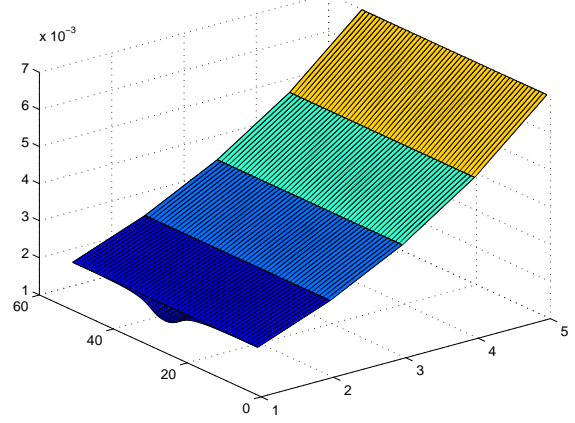

c)

FIG. 6.8. Analysis of the scattered data at the left $\Gamma_{l}$ and right $\Gamma_{r}$ boundaries of the $G_{F E M}$ domain: a) scattered data $\psi(x, s), x \in \Gamma_{l}$ superimposed with the scattered data $\psi(x, s), x \in \Gamma_{r}$ : one can see that the dent is very small. The data are scattered from the belt modeling and explosive, see Figure 6.2 Here, $c=3.2$ inside the belt and $c=1$ at all other points of $G_{F E M}$; b) homogeneous data $\psi(x, s)$ with $c=1$ in $G_{F E M}$; c) Superimposed data of a) and b). 


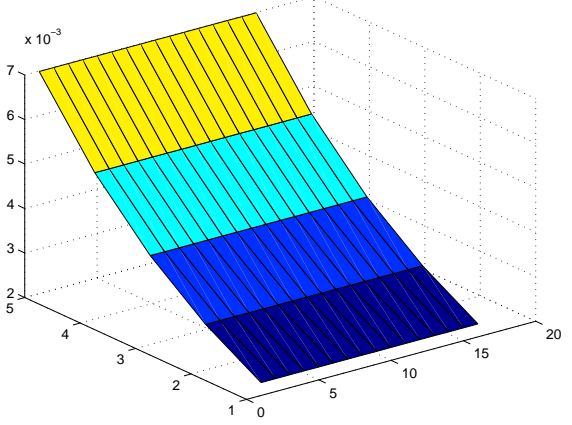

a)

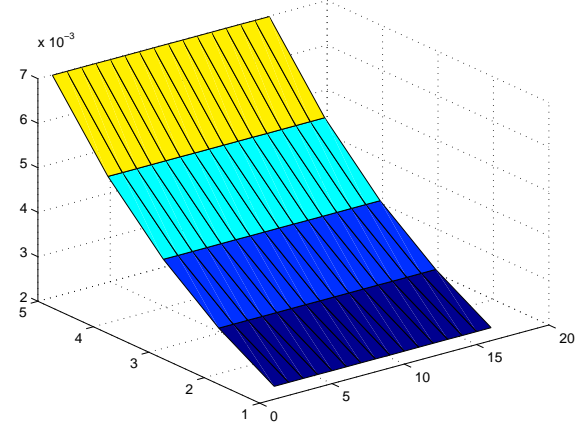

b)

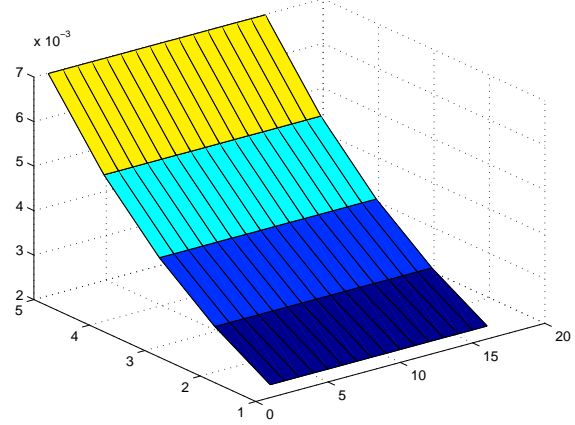

c)

Fig. 6.9. Analysis of the scattered data at the top $\Gamma_{t}$ and bottom $\Gamma_{b o t}$ boundaries of the $G_{F E M}$ domain: a) scattered data $\psi(x, s), x \in \Gamma_{t}$ superimposed with scattered data $\psi(x, s), x \in \Gamma_{b o t}$. The data are scattered from the belt with explosive of Figure 6.2 with $c=3.2$ inside the belt and $c=1$ at all other points of $G_{F E M}$; b) homogeneous data $\psi(x, s)$ with $c=1$ in $G_{F E M}$; c) Superimposed data of a) and b). 


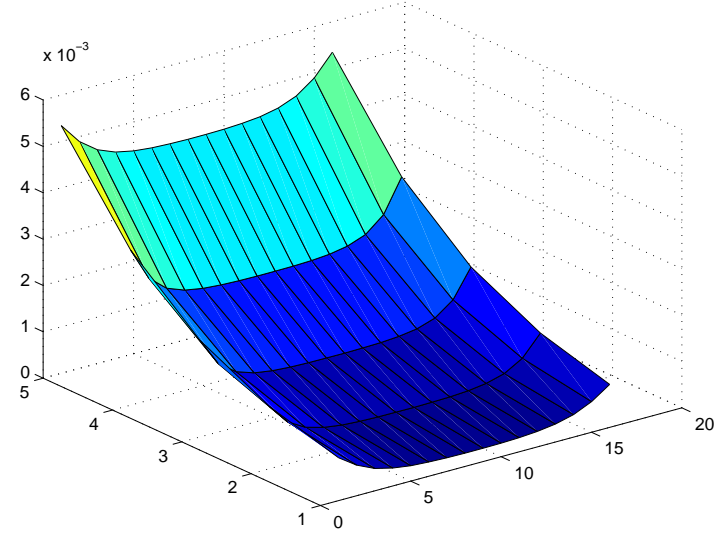

a)

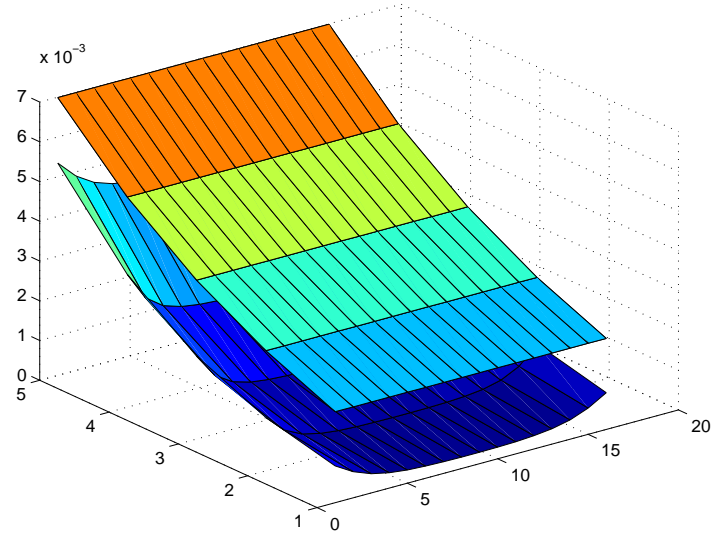

b)

FIG. 6.10. Test 5. Analysis of scattered data at the top $\Gamma_{t}$ and bottom $\Gamma_{b o t}$ boundaries of the $G_{F E M}$ : a) scattered data $\psi(x, s), x \in \Gamma_{t}$ superimposed with scattered data $\psi(x, s), x \in \Gamma_{b o t}$. b) Superimposed data of a) (low figure) and homogeneous data $\psi(x, s)$ (top figure) computed with $c=1$ in $G_{F E M}$. 

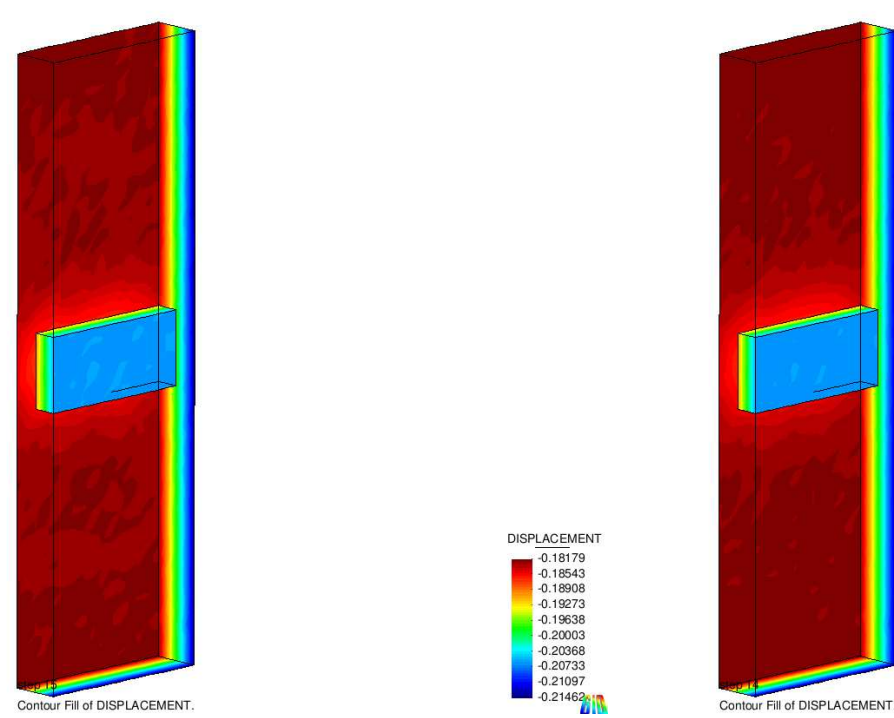

a) $s=6$
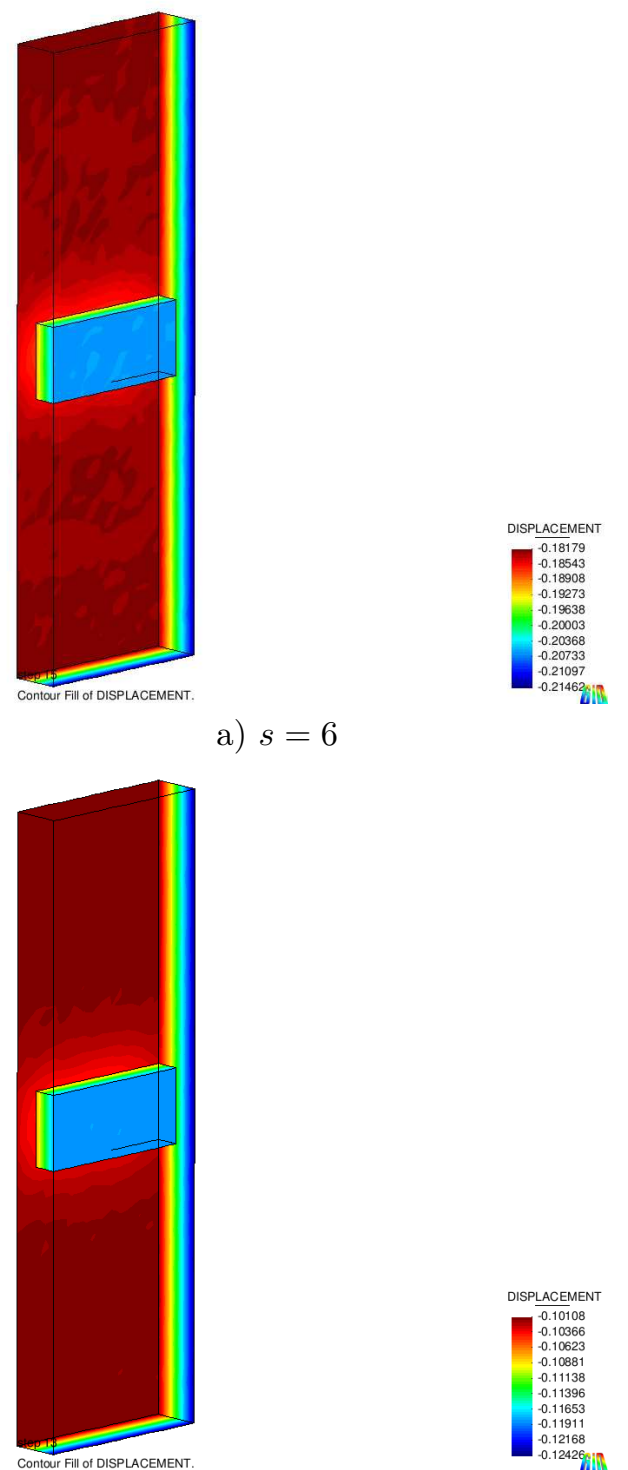

b) $s=7$
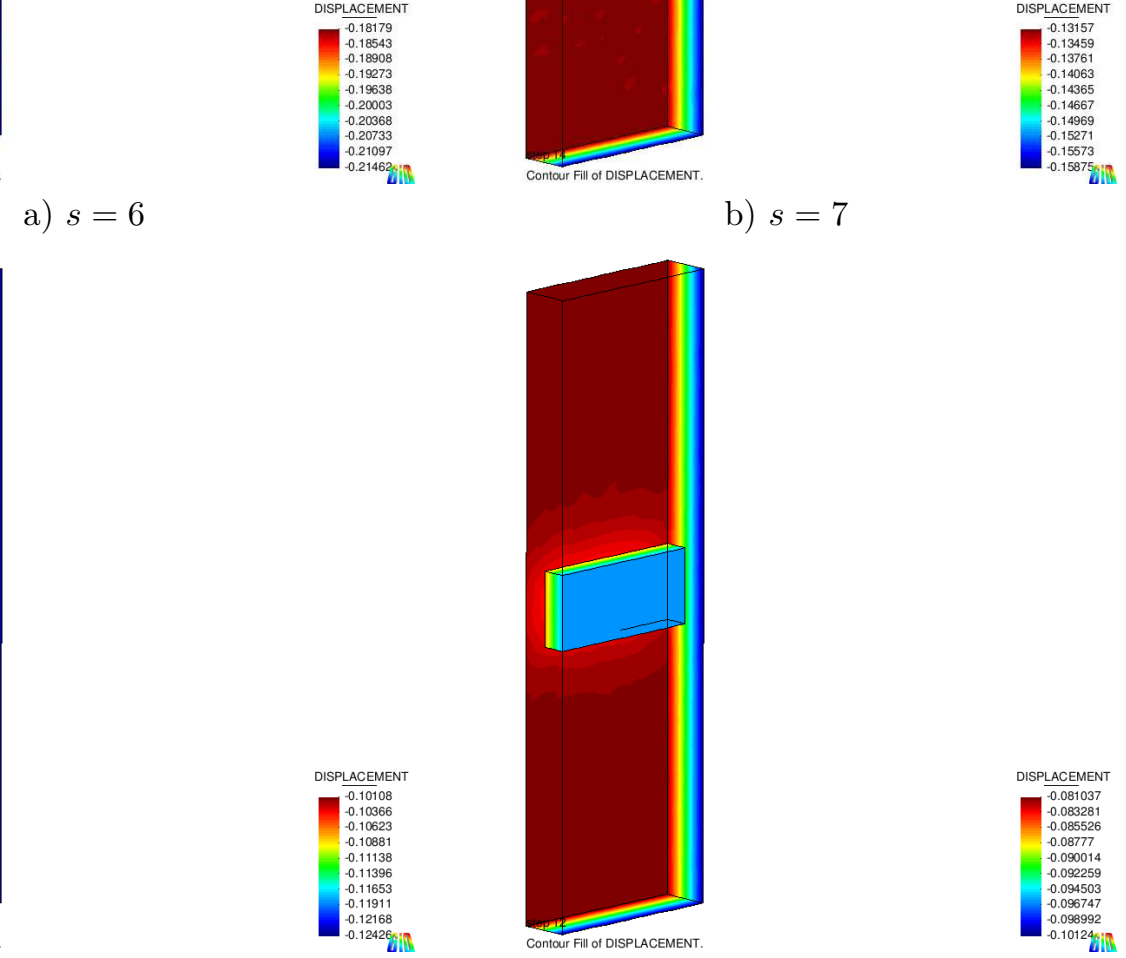

a) $s=8$

b) $s=9$

Fig. 6.11. Test 5. Backscattered data $\psi(x, s), x \in \Gamma$ immersed into data $\psi(x, s), x \in \partial \Omega \backslash \Gamma$ computed with $c=1$ in $\Omega=G_{F E M}$. Data are presented at different pseudo-frequencies $s=6,7,8,9$. 

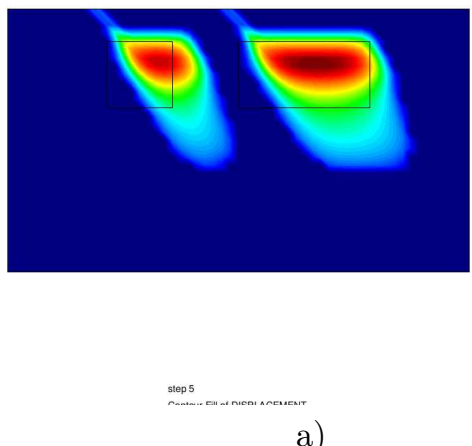

a)
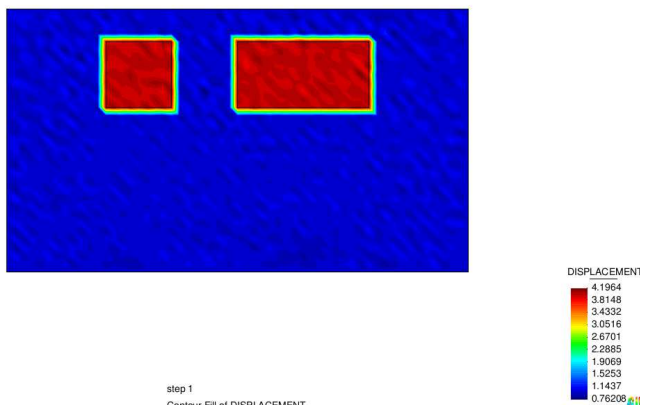

b)

FIG. 6.12. Test 1: a) The computed function $c_{3,5}(x)$. Maximal values of this function are 4.07 in both imaged mine-like targets and $c_{3,5}(x)=1$ outside of imaged targets. The image is accurate: compare with Figure 6.1 c) and with (6.20). b) The reconstructed function $c_{1,1}(x)$ for the the case when the exact tail function $V^{*}(x)$ is known. However, this is an unrealistic case, which is presented here only to demonstrate the accuracy of our method in the ideal case.

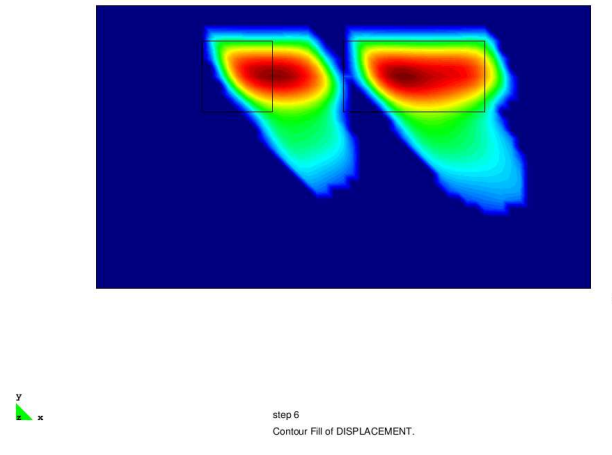

a)

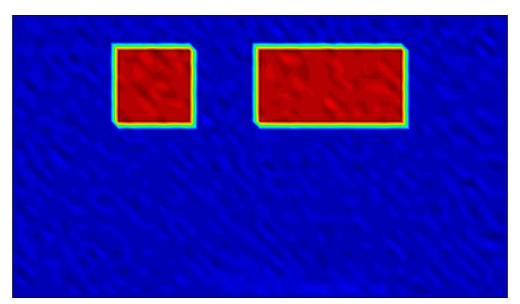

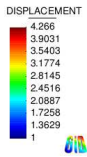

in

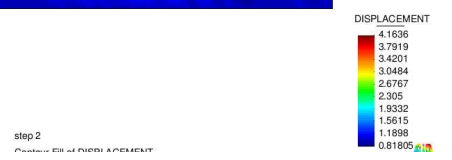

b)

Fig. 6.13. Test 2: a) The computed function $c_{8,6}(x)$. Maximal values of this function are 4.27 in both imaged mine-like targets and $c_{8,6}(x)=1$ outside of imaged targets. The image is accurate: compare with Figure 6.1 r c) and with (6.20). b) The reconstructed function $c_{1,1}(x)$ for the the case when the exact tail function $V^{*}(x)$ is known. 


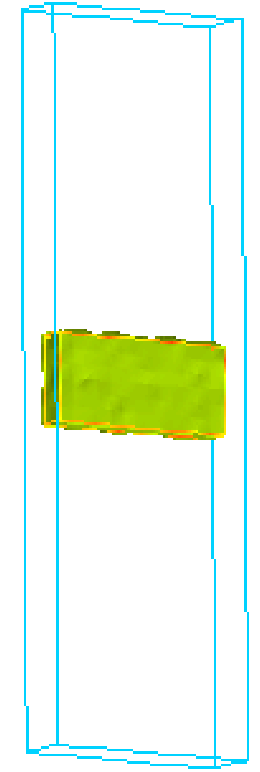

a) $\max c_{1,1} \approx 3.2$

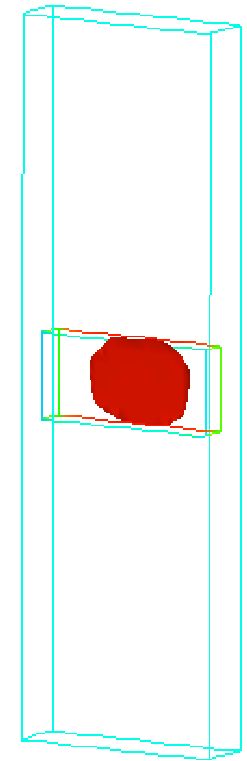

b) $\max c_{3,2} \approx 3.54$

FIg. 6.14. Test 3. Reconstruction of a belt with explosives for the case (6.24). a) The computed function $c_{1,1}(x)$ for the case when the exact tail function $V^{*}(x)$ is known. The reconstruction is perfect. However, this is an unrealistic scenario. We display it here only to show that our method is accurate in an ideal case. b) The reconstruction for the case when the initial tail function $V_{1,1}(x)$ is taken the same as the one for the uniform medium with $c(x) \equiv 1$. The computed function $c_{3,2}(x)$ is shown. Observe that $\max c_{3,2}(x)=3.54$ inside of the imaged inclusion (belt with explosives) and $c_{3,2}(x)=1$ everywhere else. The image is accurate. 


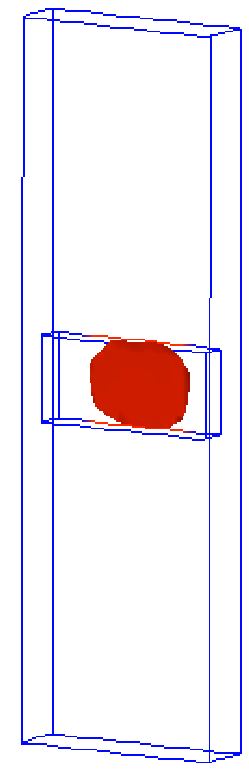

a) $\max c_{3,2} \approx 3.54$

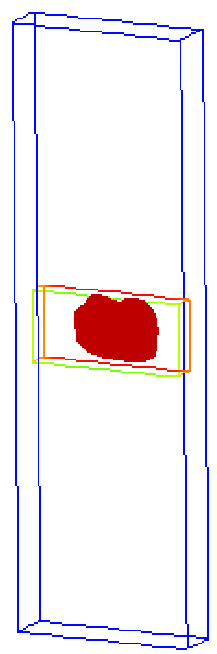

b) $\max c_{2,3} \approx 3.09$

FIg. 6.15. Reconstruction with back-scattered data. a) Test 4. The computed image of the function $c_{3,2}$. Observe that $\max c_{3,2}(x)=3.54$ inside of the imaged inclusion (belt with explosives) and $c_{3,2}(x)=1$ everywhere else. b) Test 5 . This is the most challenging test since the medium is a quite heterogeneous one, see (6.23). The computed image of the function $c_{2,3}(x)$. One can see that $\max c_{2,3}(x)=3.09$ inside of the imaged inclusion ("belt" with explosives) and $c_{2,3}(x)=1$ everywhere else. Both images are quite accurate ones. 